\title{
SHCBP1 interacting with EOGT enhanced 0- GICNAcylation of NOTCH1 and promoted the development of pancreatic cancer .
}

\section{Can Yang}

Shengli Clinical Medical College of Fujian Medical University https://orcid.org/0000-0002-1648-3452 Jianfei Hu

Shengli Clinical Medical College of Fujian Medical University

Qian Zhan

Ruijin Hospital,Shanghai Jiao Tong University School of Medicine

\section{Zu-Wei Wang}

Shengli Clinical Medical College of Fujian Medical University

Ge Li

Union Hospital,Fujian Medical University

Jing-Jing Pan

Shengli Clinical Medical College of Fujian Medical University

\section{Long Huang}

Shengli Clinical Medical College of Fujian Medical University

Cheng-Yu Liao

Shengli Clinical Medical College of Fujian Medical University

\section{Yi Huang}

Shengli Clinical Medical College of Fujian Medical University

\section{Yi-Feng Tian}

Fujian Provincial Hospital

\section{Bai-Yong Shen}

Shanghai Jiao Tong University Medical School Affiliated Ruijin Hospital

Jiang-Zhi Chen

Union Hopital,Fujian Medical University

\section{Yao-Dong Wang}

Fujian Provincial Hospital

Shi Chen ( $\nabla$ wawljwalj@163.com )

Union Hospital, Fujian Medical University

\section{Research}


Keywords: SHCBP1, EOGT, O-GIcNAcylation, NOTCH1, pancreatic cancer

Posted Date: October 27th, 2020

DOI: https://doi.org/10.21203/rs.3.rs-95104/v1

License: (c) (i) This work is licensed under a Creative Commons Attribution 4.0 International License. Read Full License 


\section{Abstract}

Background: Pancreatic cancer(PDAC) remains one of the most lethal cancers worldwide, The accumulation of many abnormal epigenetic abnormalities contribute to the fatal prognosis of PDAC,While available studies are still limited. Many studies have confirmed that the Shc SH2-domain binding protein 1(SHCBP1) plays as proto-oncogene in cancers. However, whether SHCBP1 plays a role in pancreatic cancer oncogenesis is still unknown.EGF domain-specific O-linked GlcNActransferase (EOGT) acts as a key participant in the O-GIcNAcylation of NOTCH1. EOGT mutations inhibit the NOTCH1 signalling pathway and cause several congenital developmental disorders, However, the role of EOGT in malignancies has not been reported.

Methods:SHCBP1 and EOGT were identified as proto-oncogenes in PDAC by High-throughput sequencing and Cox regression analysis,and validated by Internal and External cohort and public databases. The function of SHCBP1 and EOGT was determined in vitro and in vivo, The underlying mechanism was

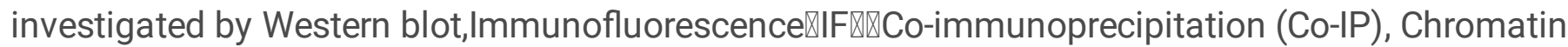
immunoprecipitation (ChIP)『and luciferase analyses.

Results: The expression of EOGT and SHCBP1 was significantly elevated and correlated with worse prognosis in PDAC patients. In vitro, SHCBP1 overexpression promoted pancreatic cancer cell proliferation,migration and invasion, while knocking down SHCBP1 and EOGT inhibited these malignant processes. In vivo data showed that SHCBP1 overexpression promoted xenograft growth and lung metastasis and shortened survival in mice,whereas knocking down either EOGT or SHCBP1 expression suppressed xenograft growth and metastasis and prolonged survival. Morever囚EOGT and SHCBP1 enhance the 0-GIcNAcylation of NOTCH1, subsequently promoting the nuclear localization of the Notch intracellular domain (NICD) and inhibiting the transcription of E-cadherin and P21 in pancreatic cancer cells.

Conclusion:All in allखThis research revealed that SHCBP1 and EOGT act as proto-oncogenes in PDAC by enhancing the O-GIcNAcylation of NOTCH1『Which provides a new field of vision for new therapeutic targets for pancreatic cancer.

\section{Background}

Pancreatic cancer is one of the most lethal cancers worldwide, and the 5-year survival rate of advanced PDAC has been less than $5 \%$ for the past few decades despite advances in surgery and adjuvant treatment methods [1]. The accumulation of many abnormal epigenetic abnormalities contribute to the fatal prognosis of PDAC,While available studies are still limited [2]. Therefore, exploring the mechanisms involved in tumorigenesis and identifying new chemotherapeutic strategies areimportant for enhancing the effectiveness of chemotherapy and reducing tumour recurrence.

Notch signalling is active at multiple stages during pancreatic cancer development and progression [3].The Notch pathway plays important roles in transcriptional regulation in response to extracellular 
signal transduction. The receptor-ligand interaction induces sequential cleavage of the Notch receptor and the release of the Notch intracellular domain (NICD), subsequently leading to its nuclear localization [4].

Mounting evidence indicates that the release of the NICD from its ligands leads to aberrant activation of Notch signalling in a variety of malignancies. The extracellular domain of NOTCH1 contains up to 36 tandem epidermal growth factor (EGF)-like repeats, which can be modified by three major types of Oglycosylation: O-fucosylation, O-glucosylation and O-GIcNAcylation (O-GIcNAc).EGF domain-specific Olinked GlcNActransferase (EOGT), a recently identified enzyme that residesin the endoplasmic reticulum (ER) andrecognizes only a limited number of target proteins (such as Notch1 receptors), adds 0-GIcNAc moieties on NOTCH1 EGF repeats to regulate ligand-induced Notch signallingactivation and vascular development in mammals [5].

EOGT mutations inhibit the NOTCH1 signalling pathway and cause several congenital developmental disorders, such as Adams-Oliver syndrome [6]. Activation of the NOTCH1 signalling pathway promotes pancreatic cancer cellproliferation, stemnessand chemotherapy resistance $[7,8]$. However, the mechanism by which O-GIcNAcylation of NOTCH1 catalysed by EOGT is involved in the progressive behaviour of pancreatic cancerremains unknown.

SHCBP1 (Shc SH2-domain binding protein 1), which was originally shown to interact with the adapter protein Shc A [9], plays an important role incell division, proliferation, differentiation, and embryonic development [10,11]. SHCBP1 is required for midbody organization and cytokinesis completion [10]. Studies have proposed that SHCBP1 promotes the cell cycle transition from G1 to S phase and confirmed that SHCBP1 is closely related to the development of breast cancer [12], lung cancer [13], prostatecancer [14] and other malignanttumours. However, whether SHCBP1 plays a role in pancreatic cancer progression has never been reported.

In the present study, we investigated the clinical significance and regulatory mechanisms of EOGT and SHCBP1 in PDAC. We identified that SHCBP1 and EOGT act as oncogenes in PDAC by promoting cell proliferation, migration, and invasion. Furthermore, we proposed that SHCBP1 and EOGT interact to mediate Notch1 0-GIcNAcylation and NICD nuclear localization, leading to transcriptional regulation during pancreatic cancer progression. These results confirm that SHCBP1/EOGT are involved in Notch1 signal transduction and suggest SHCBP1and /EOGT as potential predictive biomarkers and therapeutic targets for pancreatic cancer.

\section{Materials And Methods}

\subsection{Patients and specimens}

We collected pathological specimens from 186 patients with pancreatic cancer. All patients who underwent pancreaticoduodenectomy or distal pancreatectomy between September 2009 and September 2014 at Fujian Provincial Hospital or Fujian Union Hospital affiliated with Fujian Medical University were retrospectively enrolled in this study. We collected paraffin-embedded tissue blocks from each patient to 
construct tissue microarrays (TMAs).Detailed clinicopathological data of all the patients, including age, sex, tumour size, microvascular invasion, portal vein invasion, lymph node metastasis and tumour stage, were extracted. Patients were followed up until March 2020.We also collected 158 PDAC patients from Ruijin Hospital as external validation.All experiments involving humans were approved by the Fujian Provincial Hospital Accreditation Committee. Animal experiments were carried out in strict accordance with the programme approved by the Animal Protection and Use Committee of Fujian Medical University.

\subsection{RNA sequencing and candidate genes}

RNA high-throughput sequencing was performed in Amoydx. Briefly, the reads number obtained by RNA high-throughput sequencing was standardized to obtain the transcripts per million (TPM) value. Cox regression analysis was performed on the TPM value of the gene based on disease-free survival (DFS) to obtain high-risk genes. The screening average was TPM > 10 genes, and the top 20 genes with the highest Hazard ratio (HR) were selected as candidate genes.

\subsection{Public datasets}

Three GEO datasets(http://www.ncbi.nlm.nih.gov/geo/) were downloaded (GSE46234, GSE46385, and GSE71989) and analysed in this study.Gene expression and survival data were obtained from GEPIA2 (http://gepia.cancer-pku.cn/) and KMplot (http://kmplot.com/analysis/).

\subsection{Immunohistochemistry}

TMA sections were immunostained with antibodies targeting SHCBP1 and EOGT as described in previous studies[27, 28], and immunostained sectionswere blindly reviewed and scored by two pathologists. The staining intensities of SHCBP1 and EOGT were assigned as follows: 0 (negative), 1 (weak), 2 (medium) and 3 (strong), andthe positive rate of tumour cells was scored as follows: (1) (0-25\%), (2) (26-50\%), (3) $(51-75 \%)$ and (4) (76-100\%). The immune response score (IRS) was calculated by multiplying the staining intensity and positive rate scores and allowed for the staining patterns to be divided into three categories: weak (IRS: 0-6), strong (IRS: 7-12).

Human pancreatic cancer cell lines (Panc-1,Bxpc3) and a human embryonic kidney cell line (HEK-293T) were obtained from the American Type Culture Collection (ATCC, Manassas, VA, USA) and maintained in DMEM(Dulbecco's modified Eagle's medium) supplemented with $10 \%$ foetal bovine serum (FBS)at $37^{\circ} \mathrm{C}$ in a humidified chamber with $95 \%$ air and $5 \% \mathrm{CO} 2$.

\subsection{Establishment of cell lines}

Plasmids were purchased from the GeneChem Corporation (Shanghai, China). Gene transfection was conducted by using Lipofectamine3.0 (Invitrogen) in HEK-293Tcells. The lentivirus-containing supernatant was collected $48 \mathrm{~h}$ and $72 \mathrm{~h}$ after transfection and added to pancreatic cancer cell cultures according to the manufacturer's instructions. Stably infected pancreatic cancer cell lines were selected by puromycin.

\subsection{Cell proliferation assay}


Cell proliferation was assessed by a Cell Counting Kit-8 (CCK-8) kit (Cat. no. CK04; Dojindo Molecular Technologies, Inc., Kumamoto, Japan). Cellswere cultured in 96 -well plates for 1-5 days, after which CCK-8 solution ( $10 \mu \mathrm{l})$ was added to each well. The number of cells was quantified by measuring the absorbance at $450 \mathrm{~nm}$ on a microplate reader. Three independent experiments were performed.

\subsection{Colony formation}

Cells were trypsinized into single-cell suspensions and seeded in 12-well plates at a density of 800 cells/well. After 10 days of culture, the cells were fixed in a $4 \%$ formaldehyde solution for 20 min, stained with $0.1 \%$ crystal violet solution, rinsed withphosphate-buffered saline (PBS), dried in air and photographed. Quantitative analysis was performed bycounting the number of colonies stained in each well. Three independent experiments were performed.

\subsection{Cell cycle assay}

A cell cycle assay kit (Cat. no. C1052) was purchased from Beyotime Institute of Biotechnology (Haimen, China). The cells were fixed with $70 \%$ ethanol at $4{ }^{\circ} \mathrm{C}$ for $24 \mathrm{~h}$ and then resuspended in ice-cold PBS before they were resuspended with a staining solution containing RNase $A$ and propidium iodide $(\mathrm{PI})$. The DNA content was assessed using a flow cytometer, and the number of cells in the different cell cycle phases was counted using ModFit version 3.2 (Verity Software House, Topsham, ME, USA). Three independent experiments were performed.

\subsection{Cell invasion and metastasis}

The wound healing experiment was conducted with PDAC or Bxpc-3 cells seeded in a 6-well culture plate and grown to $>90 \%$ confluence. After $12 \mathrm{~h}$, a wound was created by scratching a pipette tip across the monolayer, and images of cells that migrated into the wound were captured after 24 and $48 \mathrm{~h}$. In the Transwell invasion assay, cells in serum-free DMEM were seed in the upper chambers of Transwell plates, whereas $400 \mu$ of complete DMEM was added to the lower chamber. After $24 \mathrm{~h}$ of culture, the cells that migrated to the lower chamber were fixed with $4 \%$ paraformaldehyde and stained with $0.1 \%$ crystal violet. The numbers of cells on the lower chamber membranes were counted under a microscope. Three independent experiments were performed.

\subsection{Luciferase reporter assay}

Luciferase assays were performedin accordance with the manufacturer's instructions. Briefly, the Ecadherin and P21 promoters were subcloned into the pGL3-Basic luciferase expression vector (Genepharma). Panc-1 cells were plated into 12-well plates (Corning Incorporated) andtransiently cotransfected with appropriate pcDNA3.1 plasmids and luciferase plasmids using the X-tremeGENE HP DNA Transfection Reagent (Roche). Cell lysates were harvested $24 \mathrm{~h}$ later, and then luciferase activities were measured with the Dual-Luciferase Reporter System (Promega, Fitchburg, WI, USA) on a luminometer (Lumat LB 9507, EG\&G Berthold, Bad Wildbad, Germany). Firefly luciferase activities were normalized to those of the Renilla luciferase. The experiment was repeated three times. 
To assess the protein-protein interaction between EOGT and SHCBP1, we transiently transfected HEK$293 \mathrm{~T}$ cells for $24 \mathrm{~h}$ with plasmids carrying Flag-EOGT and HA-SHCBP1 cDNA. The nuclear and total cellular protein fractions were extracted for immunoprecipitation and Western blot assay as described in a previous study[28]. The antibodies used wereanti-Flag (F7425,Sigma-Aldrich), anti-HA(MMS101P,Covance), anti-EOGT,(27595-1-AP,Proteintech), anti-SHCBP1(12672-1-AP,Proteintech),Ecadherin(ab231303,Abcam), anti-P21 antibody (10355-1-AP,Proteintech), anti-CDK4(ab108357,Abcam), antiN-cadherin(ab207608,Abcam).anti-O-GlcNAc antibody (CTD110.6; Thermo Scientific [24565]) ,anti-human NOTCH1 ICD antibody (D6F11, Cell signaling),hamster anti-mouse NOTCH1 ECD antibody (8G10, Santa Cruz [sc-32756]),rabbit anti-human NOTCH1 ECD antibody (H-131, Santa Cruz [sc-9170]), rabbit antihuman NOTCH1 ICD antibody (D6F11, Cell signaling)

\subsection{Chromatin immunoprecipitation (ChIP)}

Transfected Panc-1 or Bxpc-3 cells were grown in 10-cm plates to $70-90 \%$ confluency and then harvested. Cells treated with different concentration of ZLDI-8, (Cat. No.: AO-299/41409126; Specs, Zoetermeer, Netherlands), DAPT (Cat. No.: MB5152; Meilunbio, Dalian, China) or recombinant human DLL4 protein (Cat. No. Ab 108557, UK) to suppress or activatethe NOTCH1 signaling pathway.The ChIP assay was performed with a ChIP assay kit from Millipore according to the manufacturer's protocol with antibodies specific to the NICD or IgG. The immunoprecipitated DNA samples were then amplified using quantitative PCR with primers

Primers:

E-cadherin-F:GGAGAGCGGTGGTCAAAGAG

E-cadherin-R:AGTCCTGGTCCTCTTCTCCG,

P21-F:TGTCCGTCAGAACCCATGC,

P21-R:AAGTCGAAGTTCCATCGCTC,

EOGT-F:AGAGGCGTTCACTACATCACT,

EOGT-R:GCAGCCTGAAGGACAAGATAC,

SHCBP1-F:GTGTTGTCTTGGTGAAACCTAC,

SHCBP1-R:CATTTTACCAGTTGCACACTCA.

2.13. Animal experiments

To assess tumor growth ability in vivo,A nude mouse experiment was performedBrieffly, 5-week-old BALB/c male nude mice (nu/nu, Slac Laboratory Animal Co. Ltd., Shanghai, China) different SHCBP1 or 
EOGT expressed were subcutaneously injected with tumor cells $\left(5 \times 10^{\wedge} 6\right)$ in the right axillary fossa(eight mice per group). Tumor volume was measured every 4 days and calculated volume. The mice were sacrificed at 4-5 weeks after injection, and the tumors were weighed.For the lung metastasis model, $5 \times$ $10^{\wedge} 6$ stably overexpressing SHCBP1 with or without EOGT konckdown transfected Bxpc-3 cells were injected into the tail veins of nude mice. 6 weeks later, the mice were sacrificed, and the lungs were dissected to examine the histopathological metastatic loci.ForSurvival analysis, nude mice were followed up for 10 weeks after different SHCBP1 or EOGT expressed tumor cell injection. The animal experiment was approved by Animal Welfare Committee of Fujian Medical University (Fuzhou, China).

\subsection{Statistical analysis}

Data analysis and statistics were performed with SPSS 17.0 software (SPSS, Inc., Chicago, IL, USA). Ttests and one-way analysis of variance (ANOVA) followed by Fisher's least significant difference (LSD) test were used to determine differences between groups. A P-value $<0.05$ was considered to indicate a statistically significant result.

\section{Results}

\subsection{SHCBP1 and EOGT were identified as proto-oncogenes in PDAC}

High-throughput sequencing was performed in specimens from 30 advanced pancreatic cancer specimens. The clinicopathological features of the patients are presented in (Fig. 1A). SHCBP1 and EOGT were confirmed to be associated with poor prognosis in advanced pancreatic cancer by Cox regression analysis (Fig. 1B).We furtheranalyzed the correlation between SHCBP1/EOGT and clinicopathological features by IHC staining in sequencingspecimens. Results confirmed that both SHCBP1 and EOGT were associated with theTNM stage(Fig. 1C), lymphatic metastasis (Fig. 1D),and poor prognosis of pancreatic cancer(Fig. 1E). Meanwhile, 26 PDAC tissues and 15 normal pancreatic tissues from the GEO database were downloaded. A total of 376 differentially expressed genes (DEGs), including 191 downregulated and 185 upregulated genes, were identified (Supplemental Fig. 1A), and the results showed that SHCBP1 and EOGT expression was elevated(Fig. 1F). The GEPIA2 database also confirmed that the expression of SHCBP1 and EOGT was significantly increased in pancreatic cancer (Fig. 1G). Correlation analysis declared a positive correlation between SHCBP1 and EOGT(Fig. 1H), and the KMPlotdatabase showed that high SHCBP1 and EOGTexpression was associated with poorsurvivalin pancreatic cancer(Supplemental Fig. 1B-E).Therefore, we decided to further explore the functions of SHCBP1 and EOGT in pancreatic cancer.

\subsection{SHCBP1 and EOGT co-elevated and correlated with the clinicopathological features of PDAC}


Western blotting of PDAC and peritumour tissuesrevealed that the expression of SHCBP1 and EOGT was significantly upregulated in PDAC tissues (Fig. 2A-B). RT-PCR results showed that SHCBP1 expression was positively correlated with EOGT expression in pancreatic cancer tissues (Fig. 2C).To further clarify the relationship between SHCBP1 or EOGT and the clinical characteristics of patients with pancreatic cancer,we conducted IHC staining in 186 PDAC specimens. SHCBP1 and EOGT staining were observed in tumour and peritumour tissues (Fig. 2D). The expression levels of both SHCBP1 and EOGTwere higher in PDAC samples than in peritumour samples,McNemar's test confirmed that the expression of SHCBP1 was positively correlated with that of EOGT (Fig. 2E). The increased SHCBP1 expression indicated by IHC was associated with tumour size, lymphatic metastasis, portal vein invasion, and TNM stage, while high expression of EOGT was associated withtumour size, lymphatic metastasis, and TNM stage(Table I).

The OS and DFS for patients with high SHCBP1 or EOGT expression were significantly worse than those for patients with lowSHCBP1 or EOGT expression (FigF-I.).Stratification by disease stage revealed that SHCBP1 and EOGT were associated with the prognosis of pancreatic cancer(Supplemental Fig. 2A-B). Interestingly, whenwe assessed OS of PDAC patients categorized according to SHCBP1 or EOGT expression in the internal cohort,we found that EOGT or SHCBP1 was correlated with poor prognosis only in the high SHCBP1or EOGTexpression group and not in the low expression groups(Fig. 2J-M).

To further confirm the relationship betweenSHCBP1 and EOGT expressionand their prognostic value in different populations, weevaluated their relationship in an external validation cohort and obtained similar results (Supplemental Fig. 2C-E).

\subsection{SHCBP1 promoted PDAC growth in vitro and in vivo}

Clinical data showed that the high SHCBP1 expression was correlated with tumoursize. We thus wanted to determinewhether SHCBP1 expression influences the growth of PDAC cells. We established PDAC cell lines (Panc-1 and Bxpc3) with stable overexpression or knockdown of SHCBP1 (Fig. 3A).

The resultssuggested thatknocking down SHCBP1 markedlyinhibited cell proliferation (Fig. 3B-D), while overexpressing SHCBP1 promoted the proliferation of pancreatic cancer cells (Figs. 3B and 3F-G). Flow cytometricassessment of cell cycle progression revealed that SHCBP1 knockdown resulted in G1 phase arrest (Fig. 3E); by contrast, overexpression of SHCBP1 enhanced the G1/S transition (Fig. 3H). Next, we evaluated the effect of SHCBP1 in vivo. Consistent withtheresultsin vitro, xenograft tumours with high SHCBP1 expression grew faster than did the control xenografts, whereas xenografts with downregulatedSHCBP1 expression grew more slowly than did the control xenografts(Fig. 3l,J). We also detected the levels of key regulatory proteins in the cell cycle and found that P21 expression was inhibited when SHCBP1 was overexpressed (Fig. 3K). Based on the above results, we speculated that SHCBP1 promotes the proliferation of pancreatic cancer cells.

\subsection{SHCBP1 promoted PDAC metastasis and EMT}

To investigate the role of SHCBP1 in PDAC metastasis, we tested the effect of SHCBP1 on invasion and metastasis in PDAC cell lines. Knockdown of SHCBP1 expressionsignificantly suppressed the migration 
and invasion of Panc-1 cells(Fig. 4A, C), while overexpression of SHCBP1 promoted the migration and invasion of BXPC3 cells(Fig. 4B,D).BXPC3 cells overexpressingSHCBP1 andcells transduced with empty vector were injected into nude mice via tail vein. Mice injected with SHCBP1-overexpressing cells exhibited enhanced lung metastasis and shorter survival(Fig. 4E,F). The expression of EMT markers was evaluated, and the results showed that overexpression of SHCBP1 inhibited the expression of E-cadherin and promoted the expression of vimentin, while knockdown of SHCBP1 elicited the opposite effects (Fig. 4G).

\subsection{Knockdown of EOGT inhibited the malignant potential of SHCBP1 in vivo and in vitro}

To clarify the role of EOGT in the malignant behaviourof PDAC, we knocked down EOGT in Panc-1 cells (Fig. 5A),Knockdown of EOGT inhibited proliferation and metastasis in vitro (Fig. 5B-D). Moreover, we also knocked down EOGT in SHCBP1-overexpressing Panc-1 cell lines(Fig. 5A). The results confirmed that EOGT knockdownmitigated the enhanced proliferation and metastasis by ectopic SHCBP1 (Fig. 5BD).

We also evaluated the effect of EOGT in vivo using xenograft animal models. Consistent with thein vitro results, xenografts derived from cells with EOGTknockdown grew more slowly than did the control xenografts. Similar to the in vitro assay and the prolonged survival of nude mice, EOGT knockdown in SHCBP1-overexpressingxenografts also resulted in slower growth(Fig. 5E-G).

\subsection{The SHCBP1-EOGT interaction enhanced NOTCH1 O- GIcNAcylation and promoted nuclear localization of the NICD}

To explore the underlying mechanism of SHCBP1 and EOGT in the regulation of malignant behaviour, we first cotransfected HEK-293T cells withHA-SHCBP1 and Flag-EOGT plasmids and performed immunoprecipitation and Western blots to identify potential protein-protein interactions and found that EOGT and SHCBP1 interacted with each other(Fig. 6A). We alsodetected their interaction at the endogenous protein level and confirmed that SHCBP1 interacted with EOGT(Fig. 6B). Moreover,The expression of EOGT decreased in SHCBP1 knockdown cells(Fig. 6C).Confocal immunofluorescencemicroscopy was performed, and the results showed that SHCBP1 and EOGT colocalized in pancreatic cancer cells(Fig. 6D).

The main function of EOGT is to glycosylate the NOTCH1 receptor[5], so we were curious about the role of SHCBP1 and EOGT in regulating NOTCH1 O-GICNAc glycosylation.The Western blotting results showed thatoverexpressingSHCBP1 and EOGT couldenhancedO-GlcNAc glycosylation of NOTCH1. Moreover, we also discovered that the levels of the NOTCH1 intracellular segment NICD was elevated in cells with overexpression of both SHCBP1 and EOGT(Fig. 6E). 
Additionally,Immunofluorescenceanalysisrevealed that the overexpression of EOGT and SHCBP1 promotedNICDnuclear localization. Whilethe enhancedO-GIcNAcylation of NOTCH1 and NICD nuclear localization by SHCBP1 and EOGT could be reversed by the 0-GlcNAc glycosylation inhibitorbenzyl-aGalNAc(1 mg/ml) [30](Fig. 6E,F). Based on these results, we speculated that SHCBP1 and EOGT could regulate theO-GICNAc glycosylation of NOTCH1 and promote nuclear localization of the NICD.

\subsection{SHCBP1 and EOGT regulated the malignant behaviour of PDAC via the NOTCH1 pathway}

To clarify the relationship between SHCBP1/EOGT and the NOTCH1 pathway. Weutilized DLL4(1 $\mu \mathrm{g} / \mathrm{ml})$ toactivate NOTCH1 signalling in PDAC cells with different SHCBP1/EOGT expression levels[29]. The proliferation and metastasis abilities were enhanced by DLL4 but were partially reversed by knocking down SHCBP1 or/and EOGT(Fig. 7A-D). Then, we pretreated PDAC cells with DAPT( $2 \mu M)$ and ZLDI$8(2 \mu \mathrm{M})$, the resultsof which confirmed that DAPT could significantly inhibit proliferation and metastasis regardless of whether SHCBP1/EOGT was overexpressed. Interestingly, ZLDI-8-mediated inhibition of proliferation and metastasis could be rescued by overexpressing SHCBP1/EOGT(Fig. 7E-H). Therefore, we confirmed that SHCBP1 and EOGT regulate the malignant behaviour of PDAC via the NOTCH1 pathway.

\subsection{SHCBP1 and EOGT inhibited the transcriptional activity of E-cadherin and P21 by NICD}

To investigate the mechanism by which SHCBP1 and EOGTregulates the expression of E-cadherin and P21, a luciferase reporter assay was performed. Wetransfected the E-cadherin or P21 promoter into HEK293T cells and investigated the effect of EOGT/SHCBP1 knockdown or overexpression on reporter activity.We found that the DLL4 inhibited the transcriptional activityof E-cadherin and P21, while this inhibition was reducedby knocking down SHCBP1 or/and EOGT,overexpression of EOGT/SHCBP1could inhibit the activity of the E-cadherin and P21promoters, which was reversed by treatment with DAPT and ZLDI-8.Overexpression ofthe NICDalso inhibited the activity of E-cadherin and the P21 promoter,but this effect could not be reversed by DAPT and ZLDI-8(Fig. 8A-D).

Furthermore, a ChIP assay was performed in Panc-1 and Bxpc-3 cells with antibodies specific to the NICD. PCR amplification of theChIP DNA fragments shows that knockdown of SHCBP1 or EOGT could inhibit recruitment of transcription factors to the promoters of E-cadherin and P21.By contrast, overexpression of SHCBP1 or/and EOGT enhanced the recruitment of the E-cadherin and P21 promoters by the NICD but was abrogated when either SHCBP1 or EOGT was knocked down(Fig. 8E-H).

Moreover, we tested the effect of agonists or inhibitors on NICD enrichment of the E-cadherin and P21 promoter and found that the DLL4 agent could significantly enhance NICD enrichment at the promoter; however, knockdown of SHCBP1/EOGT suppressedthis enrichment,Meanwhile, the intracellular inhibitor DAPT blocked this enrichmentindependent of SHCBP1 or EOGT expression levels. The extracellular inhibitor ZLDI-8 also inhibited enrichment of the promoter,However, when we overexpressed EOGT or 
SHCBP1, the inhibition was partially rescued(Fig. 8E-H). Thus,We further explored the influence of the NICD on promoter enrichment under different concentrations of inhibitor and found that DAPT and ZLDI8 both had a strong inhibitory effect on promoter enrichment at highconcentrations. while the inhibition by ZLDI-8was partially rescued by overexpressing EOGT or SHCBP1,Therefore,we considered SHCBP1 and EOGT inhibited the transcriptional activity of E-cadherin and P21 via the NOTCH1 pathway(Fig. 8EH).Above all,We confirmed thatSHCBP1 and EOGT inhibited the transcriptional activity of E-cadherin and P21 by NICD

\section{Discussion}

For the first time, we demonstratedthat EOGT and SHCBP1 are aberrantly upregulated inadvanced pancreatic cancer andare highly associated with poor prognosis in PDAC patients. We alsofound that both SHCBP1 and EOGT can enhance the malignant behaviour of pancreatic cancer cells. Furthermore, we elaborated that SHCBP1 is involved in EOGT-mediated Notch1 O-GlcNAc glycosylation, which promotes the cleavage and nuclear localization of the NICD, leading to transcriptional repression of target genes.

Advanced pancreatic cancer is a highly lethal disease, and most patients remain asymptomatic until the disease reaches an advanced stage [15].Only $30 \% \sim 40 \%$ of patients with advanced pancreatic cancer are eligible to undergo radical resection, and there are rare effective antitumouragents. In the current study, we found that SHCBP1 and EOGT were upregulated in advanced pancreatic cancer and correlated with poorer prognosis. Univariate analysis showed that both SHCBP1 and EOGT correlated with tumour size, lymphatic metastasis, andTNM stage. Moreover, a high level of SHCBP1was shown to predict shorter survival in patients with high EOGT expression, and both proteins colocalized in pancreatic cancer cells. Thus, we proposed that the interaction of EOGT and SHCBP1 is a potential biomarker for identifying advanced pancreatic cancer.

Previous studies have reported that SHCBP1 is aberrantly expressed in many malignant tumours and is involved in several biological processes in a variety of malignant tumours. However, whether SHCBP1 plays a role in pancreatic cancer oncogenesis is still unknown. In our study, we clarified that SHCBP1 acts as an oncogene in pancreatic cancer by promoting tumour cell proliferation and metastasis. Notably, SHCBP1 promoted the G0/G1 to S phase transition in pancreatic cancer cells, while silencing SHCBP1 caused cell cycle arrest.

SHCBP1 is involved in the regulation of signalling pathways, such as the FGF, NF-KB, MAPK/ERK, $\mathrm{PI} 3 \mathrm{~K} / \mathrm{AKT}$, TGF- $\beta 1 / \mathrm{Smad}$ and $\beta$-catenin signalling pathways, and participates in T cell development, the regulation of downstream signal transduction pathways, and cytokinesis during mitosis and meiosis [16]. However, we demonstrated that SHCBP1 involved in Notch1signaling and enhanced the metastatic and invassive ability and EMT process of pancreatic cancer cells, replenishing the mechanism of SHCBP1involved tumogenesis. 
The NOTCH1 pathway plays an important role in the development of malignant tumours [17]. Notch1 receptors and ligands, as well as Notch 1 target genes, are frequently overexpressed in pancreatic cancer cells, suggesting that Notch activity is associated with the development and progression of the disease $[18,19]$. Inhibition of Notch1 signallingblocks the malignant progression of pancreatic cancer cells [20]. For the first time, we found that Notch1 signalling was activated by the interaction of SHCBP1 and EOGT, which further enhanced the progressive behaviour of pancreatic cancer cells.

Aberrant proteinO-GIcNAcylationhas been identified as a key pathological feature ofincreasing numbers of human disorders, including PDAC [21]. EOGT transfers O-GICNAc to a consensus site in EGF repeats in a limited number of secreted and membrane proteins, such as Notch1 receptors [5]. Previousstudies have confirmed that EOGT deficiencyimpairs the O-GICNAcylation of NOTCH1, leading to congenital disease [22], whereas the role of EOGT in the O-GICNAcylation of NOTCH1 has not been reported in malignancies. In our research, we found that coexpression of SHCBP1 and EOGT could promote the 0-GIcNAcylation of Notch1 and NICD production. Moreover, we also observed increased nuclear localization of the NICD, which could be inhibited by the O-GICNAcylation inhibitor. Research has confirmed that in EOGT-deficient cells, the binding of the canonical Notch ligands DLL1 and DLL4, was reduced [5],It seems equivocal that overexpression of EOGT and SHCBP1 could still activate Notch1 signalling in the presence of a low dose of the extracellularinhibitor ZLDI-8, a novel ADAM-17inhibitor which could block Notch1 signal transduction from extracellular domain to intracellular domain[26]. while the signal was inactivated in response to a high dose of ZLDI-8. We speculated that EOGTamplificated Notch1 signal transduction in response to a low dose of inhibitor.More mechanisms still need to be explored.The NICD is the ultimate functional molecule of NOTCH1. The NICD iscleaved from the plasma membrane when NOTCH1 isactivated and translocates to the nucleus to further regulate target gene transcription [23]. Previous studies reported a negative correlation between NICD levels and P21/E-cadherin [24, 25]. In our study, increased levels of NICDinhibited the transcription of P21 and E-cadherin. Moreover, SHCBP1 and EOGT enhanced the effect of the NICD.

\section{Conclusion}

In summary, our currentfindingsare the first evidence that SHCBP1 and EOGT are proto-oncogenes in PDAC development. Mechanistically, the EOGT-SHCBP1 complex regulates the 0-GIcNAc glycosylation of NOTCH1, promoting NICD nuclear localization andfurther regulating the transcription of E-cadherin and p21. Moreover, elevated SHCBP1 and EOGT expressionlevels are correlated with poor prognosis in patients with PDAC. Thus, SHCBP1 and EOGT might be potential predictors and therapeutic targets for PDAC.

\section{Abbreviations}

SHCBP1

Shc $\mathrm{SH} 2$-domain binding protein 1;

EOGT 
EGF domain-specific O-linked GlcNActransferase;

NICD

Notch intracellular domain;

DMEM

Dulbecco's Modified Eagle's Medium;

FBS

Fetal bovine serum; GEO:Gene Expression Omnibus;

HR

Hazard ratio;

CCK-8

Cell counting kit 8;

$\mathrm{IHC}$

Immunohistochemistry;

OS

Overall survival;

DFS

Disease free survival

PDAC

Pancreatic ductal carcinoma;

TCGA

The Cancer Genome Atlas.

\section{Declarations}

\section{Acknowledgements}

We thank Dr. Yi Huang for permitting us to carry out our experiments the Center for experimental Research in Clinical Medicine for this study. We are grateful to Yin Lin at the pathological department of the Fujian Provincial Hospital for the assessment of immunostaining score.

\section{Authors' contributions}

Shi Chen $\varangle$ Yao-Dong Wang , Jiang-Zhi Chen designed the study. Can Yang performed the study and wrote the paper. Jian-Fei Hu participated in data analysis. Zu-Wei Wang collected the tissues. All authors read and approved the final manuscript. No potential conflicts of interest were disclosed.

\section{Funding}

This work was supported by the High-level hospital foster grants from Fujian Provincial Hospital (\#2019HSJJ13 to S. Chen)the Natural Science Foundation for Distinguished Young Scholars of Fujian Province (\#2018J06020 to S. Chen),Education and Scientific Research Foundation of Fujian Province (\#2060402 to S. Chen),Joint Funds for the innovation of science and technology, Fujian Province 
(\#2018Y9098 to S. Chen and \#2018Y9013 to J.Z. Chen)Fujian Provincial Health and Family Planning Research Medical Inovation Project(2019-cx-3 to Shi Chen) and by the National Natural Science Foundation of China (\#81772560 to Y.D. Wang and \#81702438 to J.Z. Chen),Startup Fund for scientific research, Fujian Medical University(2019qh2046 to CanYang). Fujian Provincial Health and Family

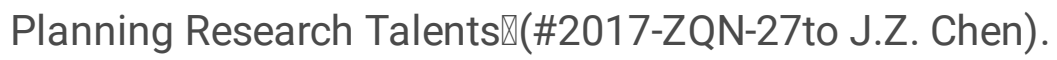

\section{Ethics approval and consent to participate}

This study was approved by the Human Research Ethics Committees of the Fujian Medical University and was carried out in accordance with the principles embodied in the Declaration of Helsinki. All in vivo animal experiments were approved by the Committee on the Ethics of Animal Experiments of Fujian Medical University.

\section{Availability of data and materials}

All data in our study are available upon request.

\section{Consent for publication}

All authors agree to the publication of our work to Journal of Experimental \& Clinical Cancer Research.

\section{Competing interests}

The authors declare that they have no competing interests.

\section{References}

[1] R.L. Siegel, K.D. Miller, A. Jemal, Cancer statistics, 2018, CA: Cancer J. Clin. 68 (2018) 7-30. https://doi.org/10.3322/caac.21442.

[2] M.T. Moyer, R.R. Gaffney, Pancreatic adenocarcinoma, N. Engl. J. Med. 371 (2014) 2140. https://doi.org/10.1056/NEJMc1412266.

[3] C.S. Nowell, F. Radtke, Notch as a tumour suppressor, Nat. Rev. Cancer 17 (2017) 145-159. https://doi.org/10.1038/nrc.2016.145.

[4] O. Meurette, P. Mehlen, Notch signaling in the tumor microenvironment, Cancer Cell 34 (2018) 536548. https://doi.org/10.1016/j.ccell.2018.07.009.

[5] S. Sawaguchi, S. Varshney, M. Ogawa, Y. Sakaidani, H. Yagi, K. Takeshita, T. Murohara, K. Kato, S. Sundaram, P. Stanley, T. Okajima, O-GIcNAc on NOTCH1 EGF repeats regulates ligand-induced Notch signaling and vascular development in mammals, Elife 6 (2017) e24419. https://doi.org/10.7554/ eLife.24419. 
[6] K.C. Schröder, D. Duman, M. Tekin, D. Schanze, M. Sukalo, J. Meester, W. Wuyts, M. Zenker, AdamsOliver syndrome caused by mutations of the EOGT gene, Am. J. Med. Genet. A 179 (2019) 2246-2251. https://doi.org/10.1002/ajmg.a.61313.

[7] Z. Zhang, H. Han, Y. Rong, K. Zhu, Z. Zhu, Z. Tang, C. Xiong, J. Tao, Hypoxia potentiates gemcitabineinduced stemness in pancreatic cancer cells through AKT/Notch1 signaling, J. Exp. Clin. Cancer Res. 37 (2018) 291-291. https://doi.org/10.1186/ s13046-018-0972-3.

[8] S. Kunnimalaiyaan, J. Trevino, S. Tsai, T.C. Gamblin, M. Kunnimalaiyaan, Xanthohumol-mediated suppression of Notch1 signaling is associated with antitumor activity in human pancreatic cancer cells, Mol. Cancer Ther. 14 (2015) 1395-1403. https://doi.org/10.1158/1535-7163. MCT-14-0915.

[9] R. Schmandt, S.K. Liu, C.J. McGlade, Cloning and characterization of MPAL, a novel Shc SH2 domainbinding protein expressed in proliferating cells, Oncogene 18 (1999) 1867-1879. https://doi.org/10.1038/sj.onc.1202507.

[10] E. Asano, H. Hasegawa, T. Hyodo, S. Ito, M. Maeda, D. Chen, M. Takahashi, M. Hamaguchi, T. Senga, SHCBP1 is required for midbody organization and cytokinesis completion, Cell Cycle 13 (2014) 27442751. https://doi.org/10.4161/15384101. 2015.945840.

[11] J. Chen, F. Lai, L. Niswander, The ubiquitin ligase $\mathrm{mLin} 41$ temporally promotes neural progenitor cell maintenance through FGF signaling, Genes Dev. 26 (2012) 803-815. https://doi.org/10.1101/gad.187641.112.

[12] W. Feng, H.C. Li, K. Xu, Y.F. Chen, L.Y. Pan, Y. Mei, H. Cai, Y.M. Jiang, T. Chen, D.X. Feng, SHCBP1 is over-expressed in breast cancer and is important in the proliferation and apoptosis of the human malignant breast cancer cell line, Gene 587 (2016) 91-97. https://doi.org/10.1016/j.gene.2016.04.046.

[13] L. Liu, Y. Yang, S. Liu, T. Tao, J. Cai, J. Wu, H. Guan, X. Zhu, Z. He, J. Li, E. Song, M. Zeng, M. Li, EGFinduced nuclear localization of SHCBP1 activates $\beta$-catenin signalingand promotes cancer progression, Oncogene 38 (2018) 747-764. https://doi.org/ 10.1038/s41388- 018-0473-z.

[14] N. Xu, Y.P. Wu, H.B. Yin, S.H. Chen, X.D. Li, X.Y. Xue, X. Gou, SHCBP1 promotes tumor cell proliferation, migration, and invasion, and is associated with poor prostate cancer prognosis, J. Cancer Res. Clin. Oncol. 146 (2020) 1953-1969. https://doi.org/10.1007/s00432-020-03247-1.

[15] R. Shah, K.T. Ostapoff, B. Kuvshinoff, S.N. Hochwald, Ablative therapies for locally advanced pancreatic cancer, Pancreas 47 (2018) 6-11. https://doi.org/10.1097/mpa. 0000000000000948.

[16] G.Y. Zhang, Z.J. Ma, L. Wang, R.F. Sun, X.Y. Jiang, X.J. Yang, B. Long, H.L. Ye, S.Z. Zhang, Z.Y. Yu, W.G. Shi, Z.Y. Jiao, The role of Shcbp1 in signaling and disease, Curr. Cancer Drug Targets 19 (2019) 854-862. https://doi.org/10.2174/ 1568009619666190620114928. 
[17] N. Wang, M.Y. Li, Y. Liu, J. Yu, J. Ren, Z. Zheng, S. Wang, S. Yang, S.L. Yang, L.P. Liu, B.G. Hu, C.C. Chong, J.L. Merchant, P.B. Lai, G.G. Chen, ZBP-89 negatively regulates self-renewal of liver cancer stem cells via suppression of Notch1 signaling pathway, Cancer Lett. 472 (2020) 70-80. https://doi.org/10.1016/j.canlet.2019.12.026.

[18] Y. Chen, Z. Li, M. Zhang, B. Wang, J. Ye, Y. Zhang, D. Tang, D. Ma, W. Jin, X. Li, S. Wang, Circ-ASH2L promotes tumor progression by sponging miR-34a to regulate Notch1 in pancreatic ductal adenocarcinoma, J. Exp. Clin. Cancer Res. 38 (2019) 466-466. https://doi.org/10.1186/s13046-0191436-0.

[19] J. Lee, J. Lee, J.H. Kim, Association of Jagged1 expression with malignancy and prognosis in human pancreatic cancer, Cell. Oncol. (2020). https://doi.org/10.1007/ s13402-020-00527-3.

[20] K. Song, Q. Li, Y.B. Peng, J. Li, K. Ding, L.J. Chen, C.H. Shao, L.J. Zhang, P. Li, Silencing of hHS6ST2 inhibits progression of pancreatic cancer through inhibition of Notch signalling, Biochem. J. 436 (2011) 271-282. https://doi.org/10.1042/bj20110297.

[21] X. Yang, K. Qian, Protein O-GlcNAcylation: emerging mechanisms and functions, Nat. Rev. Mol. Cell Biol. 18 (2017) 452-465. https://doi.org/10.1038/nrm.2017.22.

[22] A.B. Stittrich, A. Lehman, D.L. Bodian, J. Ashworth, Z. Zong, H. Li, P. Lam, A. Khromykh, R.K. Iyer, J.G. Vockley, R. Baveja, E.S. Silva, J. Dixon, E.L. Leon, B.D. Solomon, G. Glusman, J.E. Niederhuber, J.C. Roach, M.S. Patel, Mutations in NOTCH1 cause Adams-Oliver syndrome, Am. J. Hum. Genet. 95 (2014) $275-284$. https://doi.org/10.1016/j.ajhg.2014.07.011.

[23] Z. Liu, E. Brunskill, B. Varnum-Finney, C. Zhang, A. Zhang, P.Y. Jay, I. Bernstein, M. Morimoto, R. Kopan, The intracellular domains of Notch1 and Notch2 are functionally equivalent during development and carcinogenesis, Development 142 (2015) 2452-2463. https://doi.org/10.1242/dev.125492.

[24] H. Zhou, L. Gao, Z.H. Yu, S.J. Hong, Z.W. Zhang, Z.Z. Qiu, LncRNA HOTAIR promotes renal interstitial fibrosis by regulating Notch1 pathway via the modulation of miR-124, Nephrology 24 (2019) $472-480$. https://doi.org/10.1111/nep.13394.

[25] D.H. Luo, Q. Zhou, S.K. Hu, Y.Q. Xia, C.C. Xu, T.S. Lin, Y.T. Pan, J.S. Wu, R. Jin, Differential expression of Notch1 intracellular domain and p21 proteins, and their clinical significance in gastric cancer, Oncol. Lett. 7 (2013) 471-478.https://doi.org/10.3892/ol. 2013.1751.

[26] Lu H Y , Chu HX , Tan Y X, et al. Novel ADAM-17 inhibitor ZLDI-8 inhibits the metastasis of hepatocellular carcinoma by reversing epithelial-mesenchymal transition in vitro and in vivo[J]. Life ences, 2020, 244:117343. https://doi.org/10.1016/ j.Ifs.2020.117343

[27] T. Hamidi, C.E. Cano, D. Grasso, M.N. Garcia, M.J. Sandi, E.L. Calvo, et al., Nupr1- aurora kinase A pathway provides protection against metabolic stress-mediated autophagic-associated cell death, Clin. 
Cancer Res. 18 (2012) 5234e5246. https://doi.org/

10.1158/1078-0432.CCR-12-0026

[28] S. Chen, J. Chen, Q. Zhan, Y. Zhu, H. Chen, X. Deng, et al., H2AK119Ub1 and H3K27Me3 in molecular staging for survival prediction of patients with pancreatic ductal adenocarcinoma, Oncotarget 5 (2014) 10421.

[29] Tang D , Yan T , Zhang J , et al. Notch1 Signaling Contributes to Hypoxia-induced High Expression of Integrin $\beta 1$ in Keratinocyte Migration[J]. Scientific Reports, 2017, 7:43926.

https://doi.org/10.18632/oncotarget.2126

[30] AlkholiefM , Campbell R B . Investigating the role of mucin in the delivery of nanoparticles to cellular models of human cancer disease: an in vitro study[J]. Nanomedicine, 2016:1291-1302.

https://doi.org/10.1016/j.nano.2016.01.007

\section{Tables}

Due to technical limitations, table 1 is only available as a download in the Supplemental Files section.

\section{Figures}


A

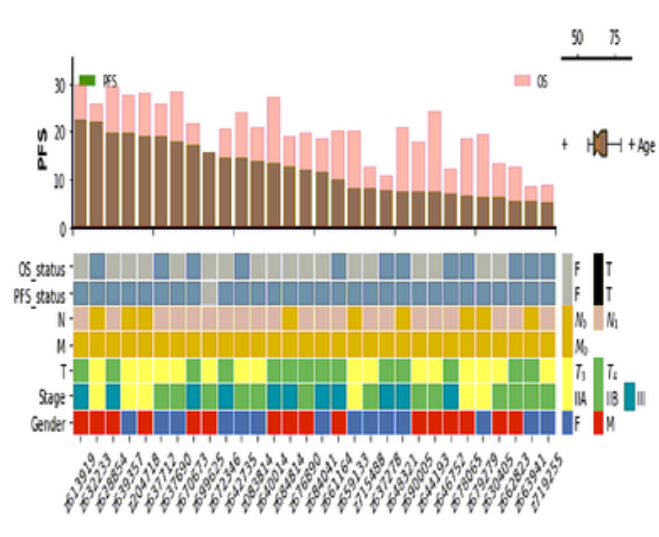

C

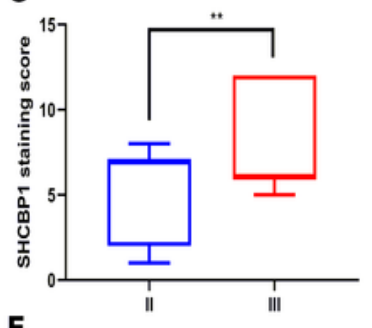

E

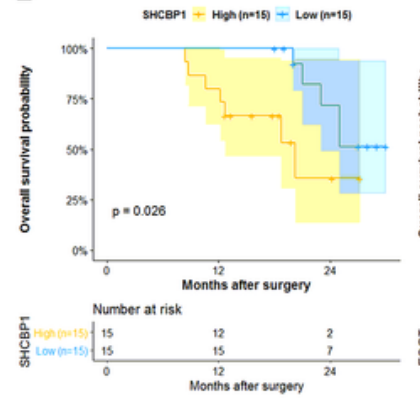

G

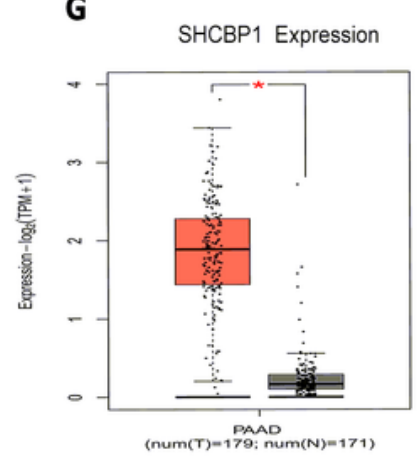

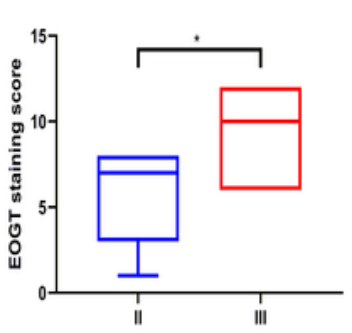

EOGT + High $(n+19)+\operatorname{Low}(\ln -19)$

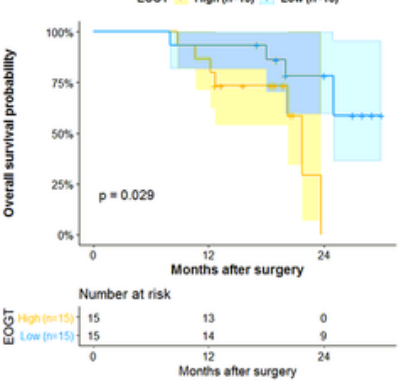

OS (median) 20.2

Age (median) 63.5

B

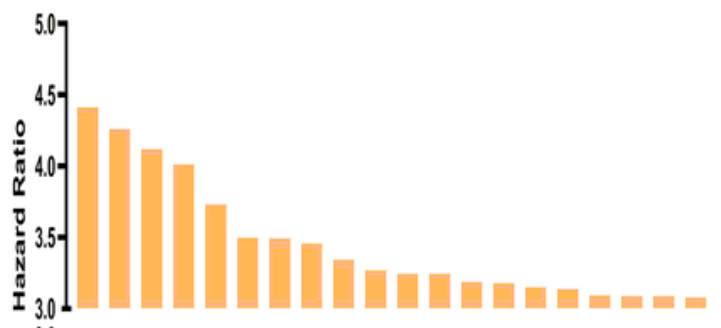

3.05
$2.0-5$
0.0

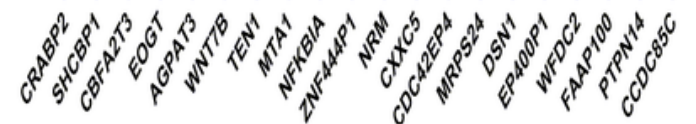

D
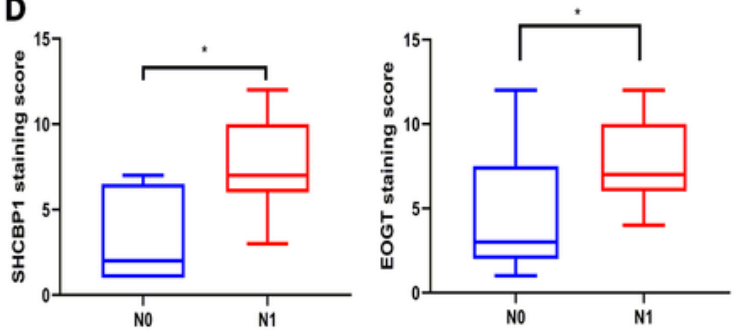

F SHCBP1 Expression

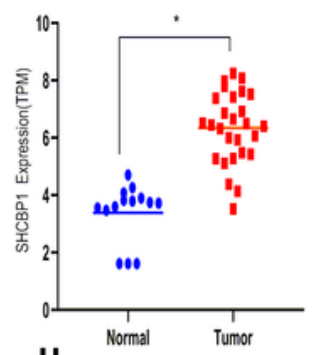

EOGT Expression

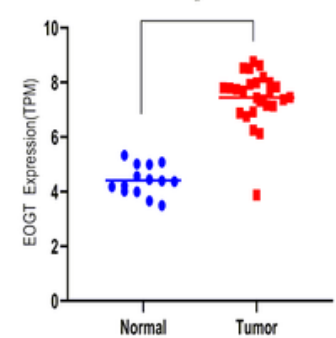

H

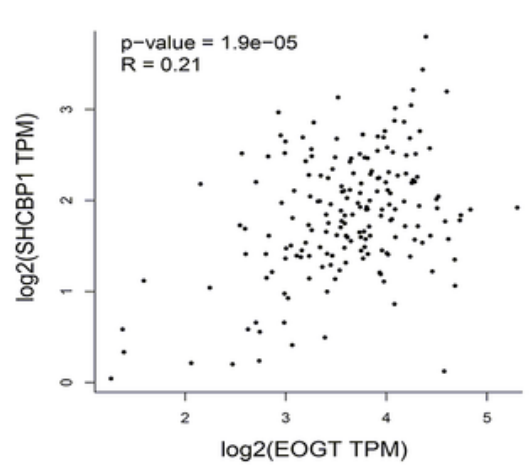

\section{Figure 1}

SHCBP1 and EOGT are important for pancreatic cancer. A.Clinicopathological features of the 30 advanced PDAC patients are presented.B.Based on DFS, UnivariateCox regression analysis was performed on the TPM values of the genes to obtain the high-risk gene group. The screening average was TPM>10per gene group, and the top 20 genes with the highest HR were selected as candidate genes.C,D.Correlation between the expression of SHCBP1/EOGT and TNM stage( C),and lymphatic 
metastasis(D).E.Comparison of OSwith different levels of SHCBP1 and EOGT expression.F,G.Expression of SHCBP1 and EOGT in pancreatic cancer tumourand adjacent normal tissues from the GEO(F)database,and GEPIA2 database $₫ \mathrm{G} \otimes, \mathrm{H}$. Correlation between the expression of SHCBP1 andEOGT from the GEPIA database.
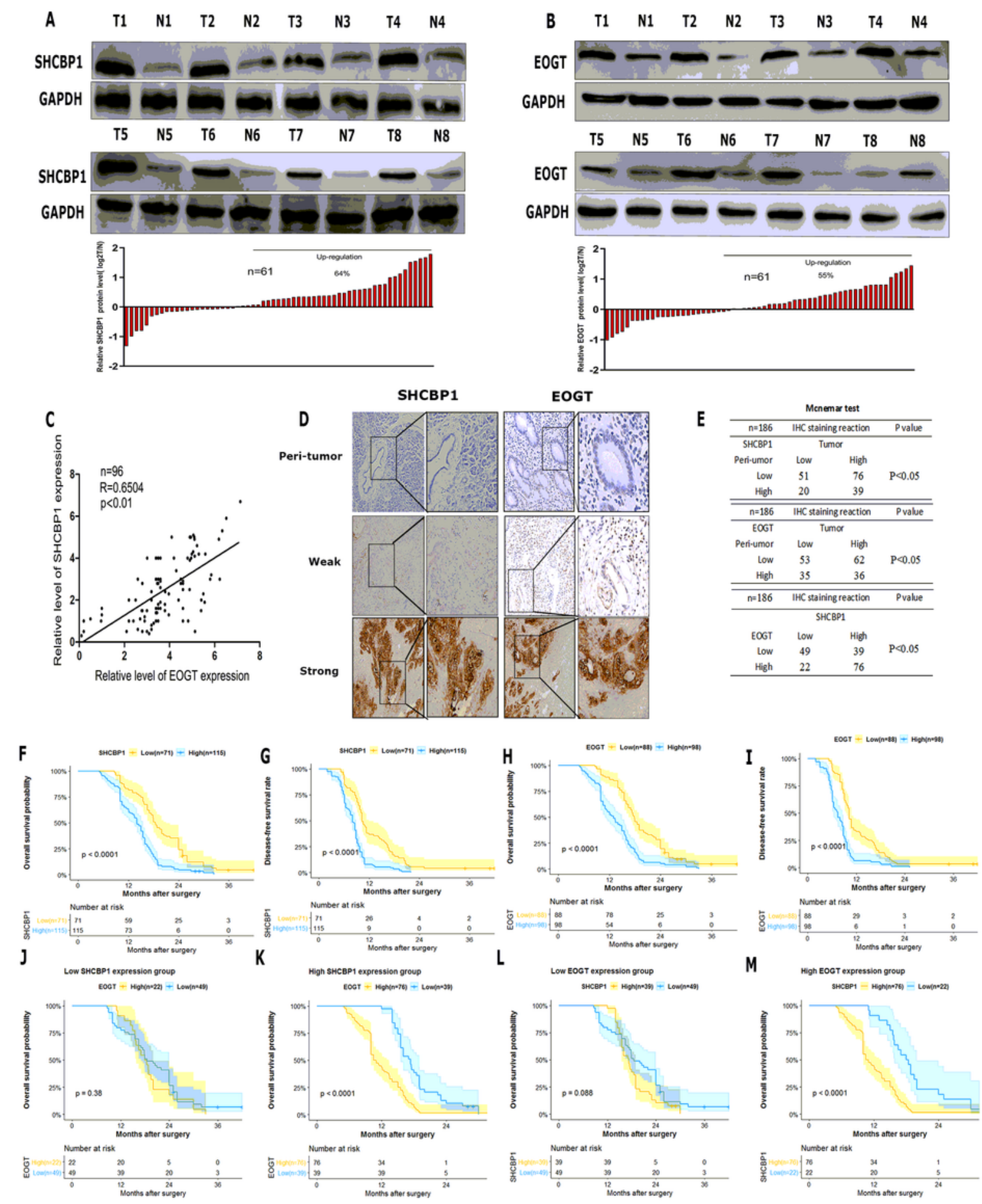

Figure 2 
The expression and prognostic value of SHCBP1/EOGT in PDAC. A,B.Representative blot images of SHCBP1and EOGT protein levels in pancreatic tumour and peritumourtissues. The $T / N$ ratios of the SHCBP1and EGOT protein levels in pancreatic tumour and peritumour tissues were presented at the bottom.C. Correlation between the expression of SHCBP1 and that of EOGTwas measured by qRTPCR.D.IHC assay of SHCBP1 (left) and EOGT (right)expression in paired human pancreatic cancer specimens.E.IHC statistical results of paired human pancreatic cancer specimens are shown,The top represents SHCBP1, the middle represents EOGT, and the bottom represents the correlation.F-I. Comparison of OS $(F, H)$ and DFS $(G, I)$ of patients with different levels of SHCBP1 and EOGT expression. (J-M).The relationship between SHCBP1 (EOGT) and OS of PDAC patients categorized according to EOGT(SHCBP1) expressionis shown. 
A

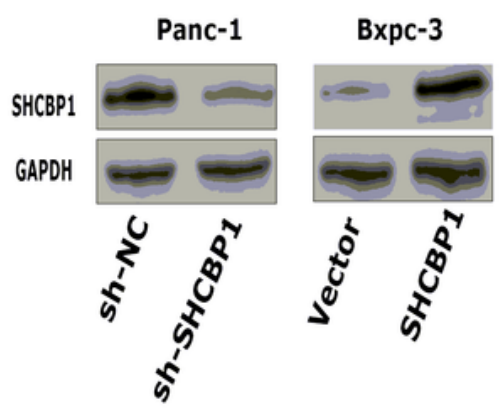

C

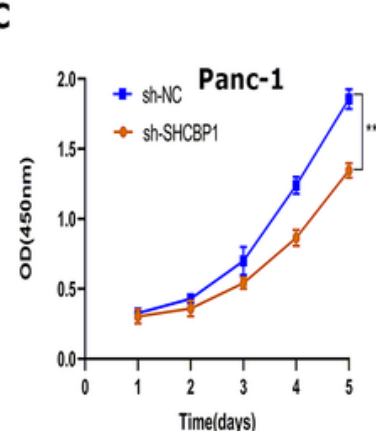

F

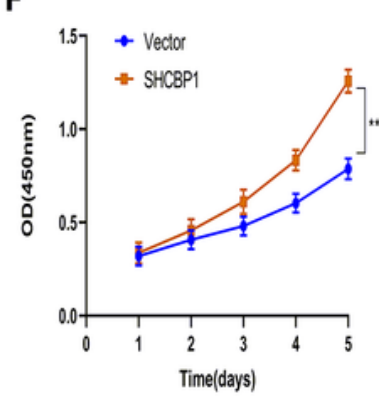

I

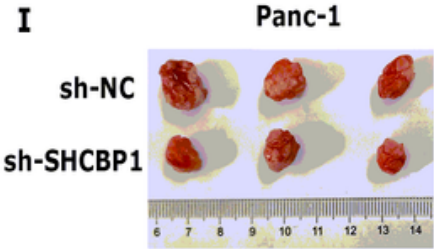

J

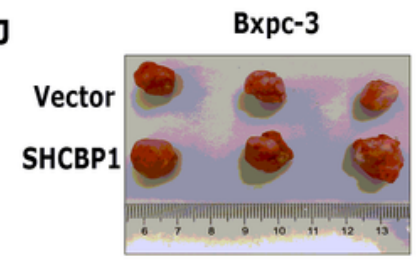

D sh-NC

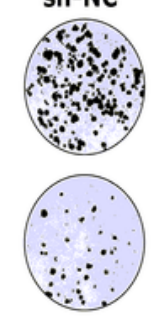

sh-SHCBP1

G Vector

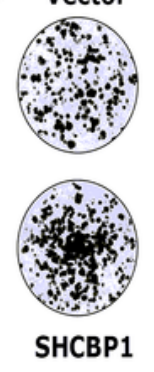

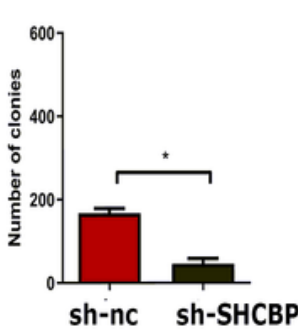

B $\rightarrow$ BXPC-3 vector

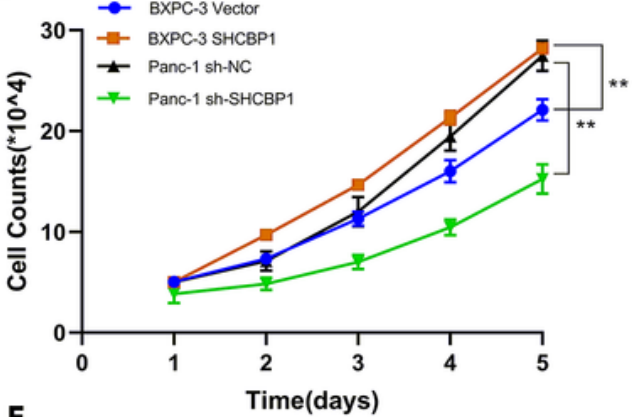

E
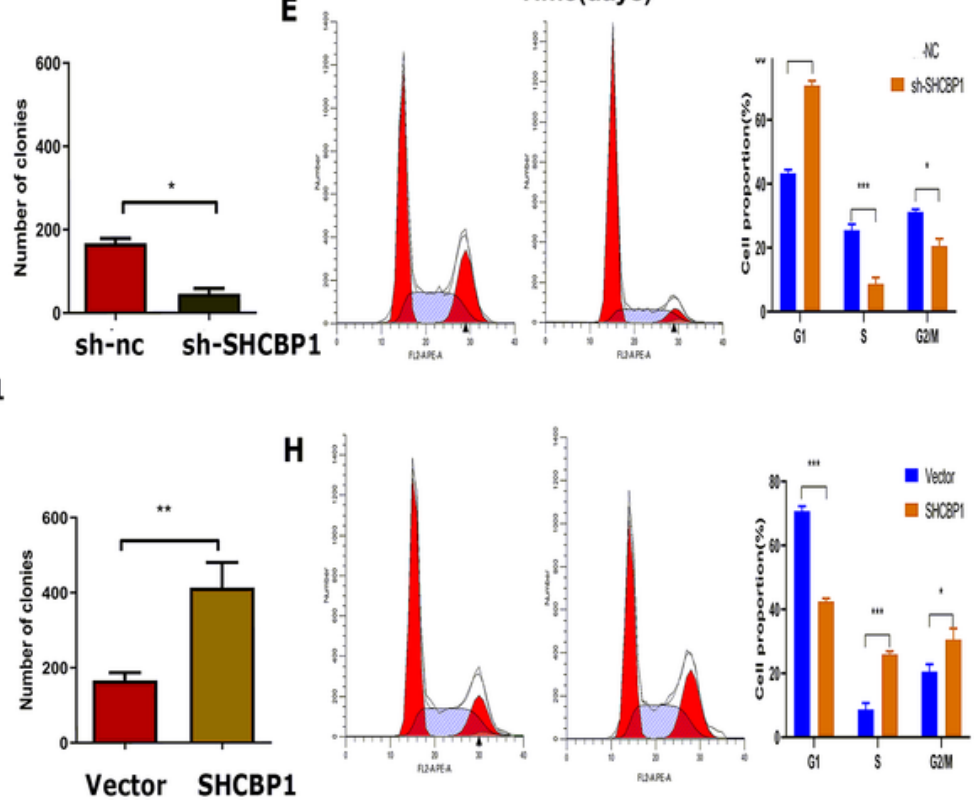

H

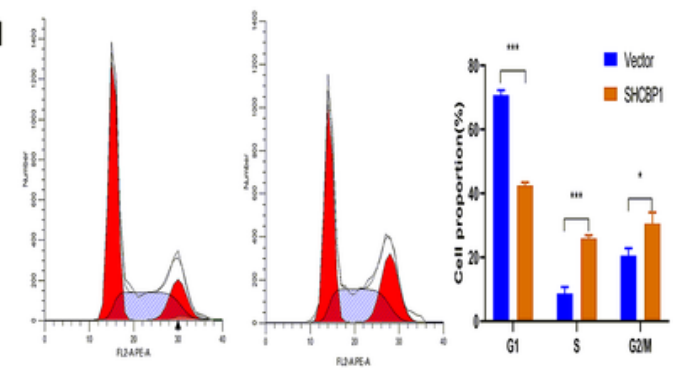

K
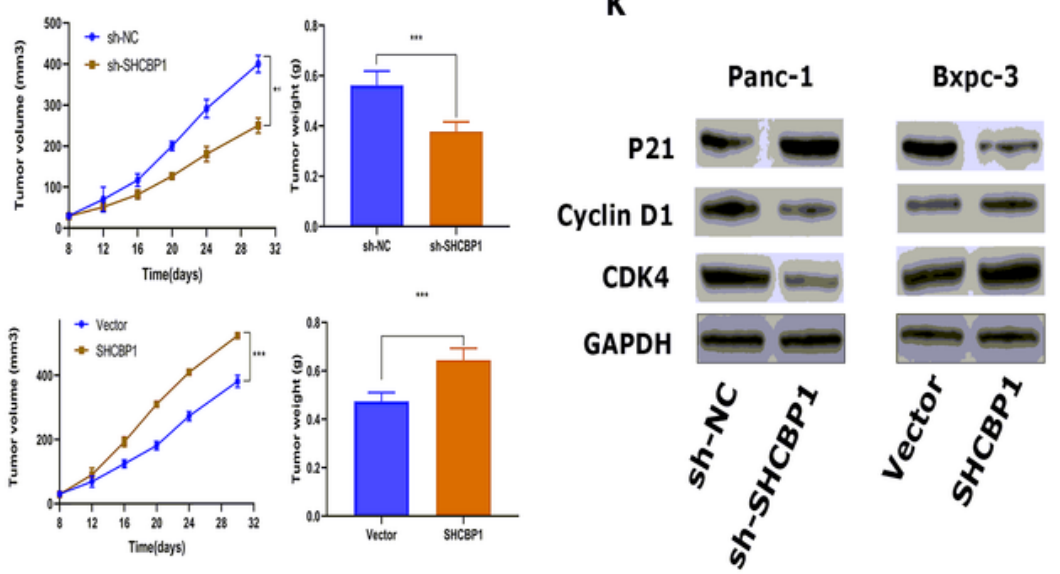

\section{Figure 3}

SHCBP1 promotes PDAC growth in vitro and in vivo. A. Panc-1 cells with SHCBP1 knockdown and Bxpc-3 cells with stable SHCBP1 overexpression were established. The changes in SHCBP1 protein expression were confirmed by Western blotting. B-D, F, GThe proliferative ability of stably transfected Panc1 or Bxpc3 cells was investigated via cell counting, CCK-8 and colony formation assays. E, H.Flow cytometry analysis of stably transfected Panc1 or Bxpc-3 cells was performed. Representative imagesof the cell 
cycle distributionare presented.I.SHCBP1 knockdown inhibitedPDAC growth in a subcutaneousxenograft model (nude mouse). The tumour volume was measured at the indicated time points $(* \star P<0.01)$. Tumours were extracted and weighed after the mice were sacrificed ( $\left.{ }^{\star \star *} \mathrm{P}<0.001\right)$.J. Overexpression of SHCBP1promotesPDAC growth in a subcutaneousxenograft model (nude mouse). The tumour volume was measured at the indicated time points $(* \star * P<0.001)$. Tumours were extracted and weighed after the mice were sacrificed ( $\left.{ }^{* \star *} \mathrm{P}<0.001\right)$. K.The changes in $\mathrm{P} 21$,CyclingD1,CDK4 protein expression were confirmed by Western blotting.

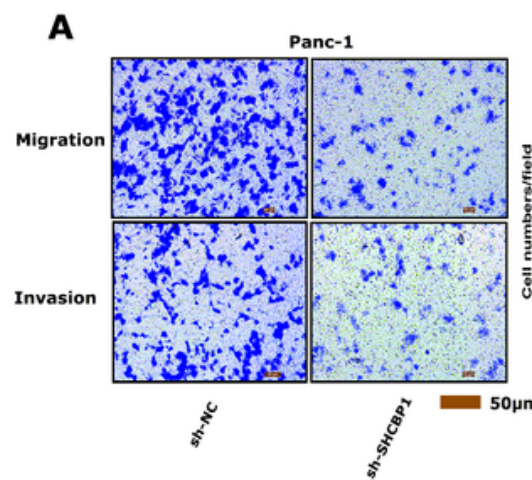

C
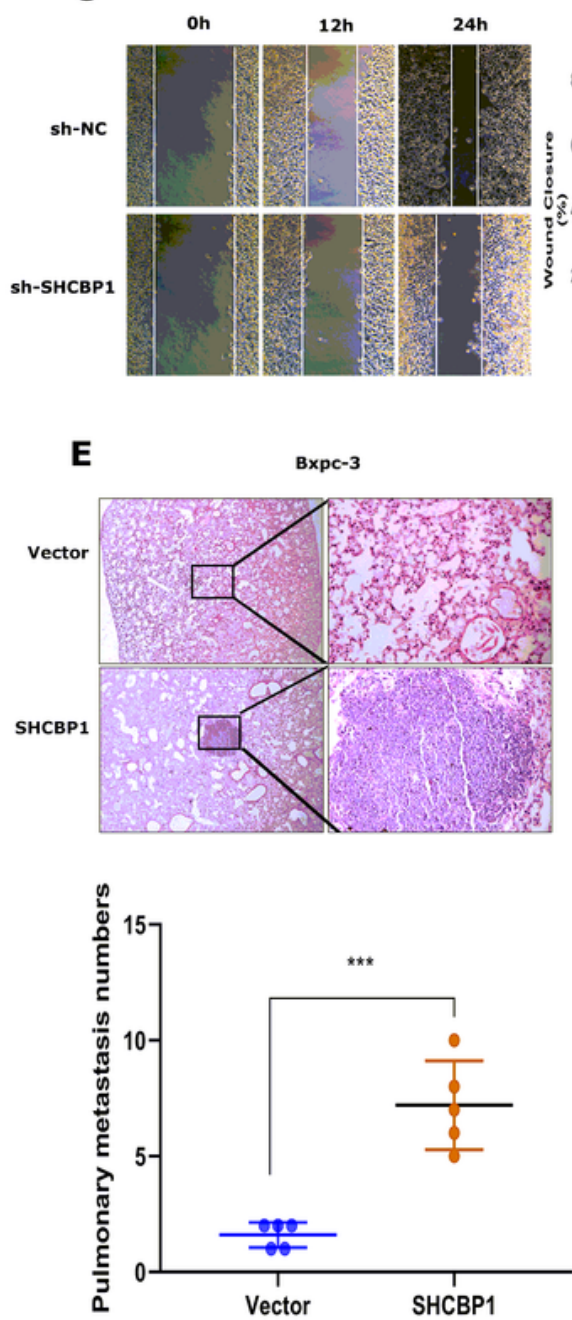

B

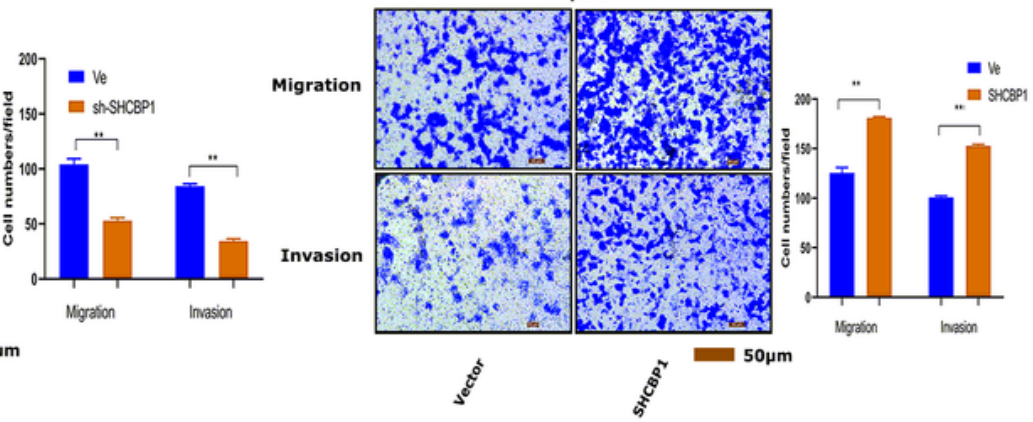

D
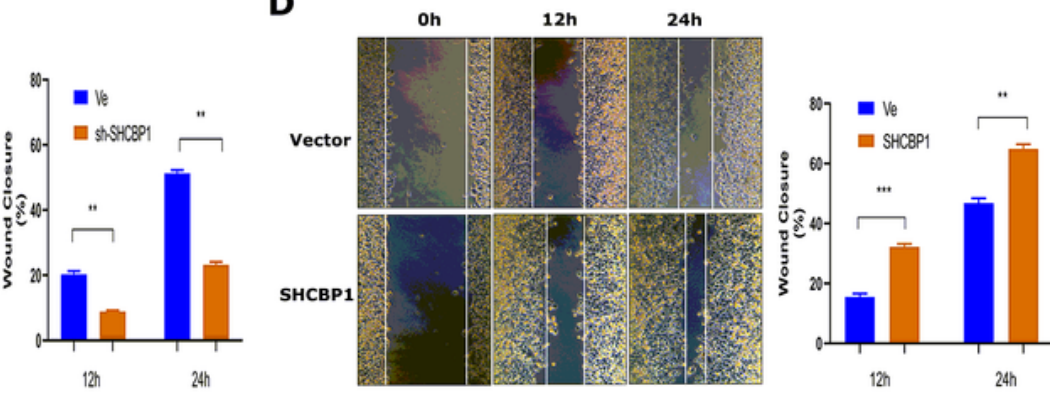

$\mathbf{F}$

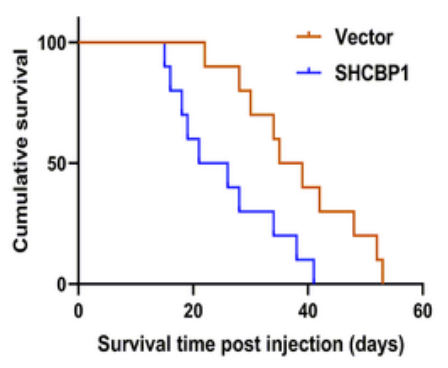

$\mathbf{G}$
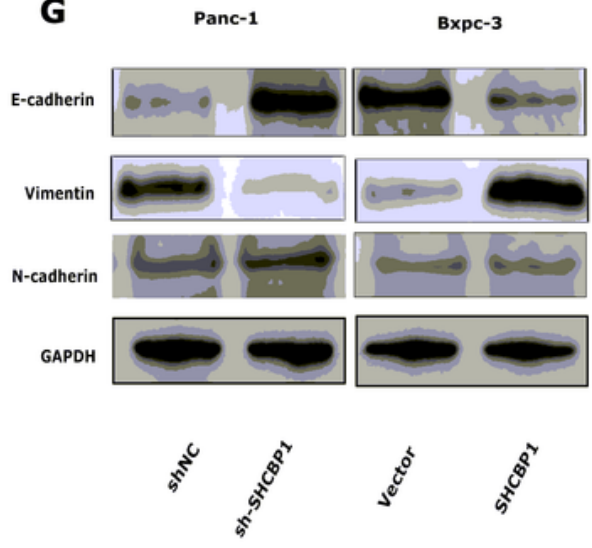


\section{Figure 4}

SHCBP1 promotes PDACmetastasis and EMT in vitro and in vivo. A, B.Transwell assays with stably transfected Panc-1 and Bxpc-3 cells were performed. Representative images and quantification of migration and invasion are presented $(* \star P<0.01 ; \star \star * P<0.001)$. C, D.Wound-healingassays with stably transfected Panc-1 and Bxpc-3 cells were performed. Representative images and quantification of wound closureare presented. E.Metastatic nodules were counted with a microscope and recorded.

Overexpression of SHCBP1 in Bxpc-3 cells significantly promoted the number of metastatic lesions in the lungs ( $* \star * P<0.001)$. HEstaining of pulmonary metastatic nodes is presented. F. The effect of SHCBP1 on OS of mice after intraperitoneal injection ofPanc-1 cells.G.Western blots forE-cadherin and vimentinexpression inPanc-1 and Bxpc-3 cells and stably transfected Panc-1 and Bxpc-3 cells. 
A

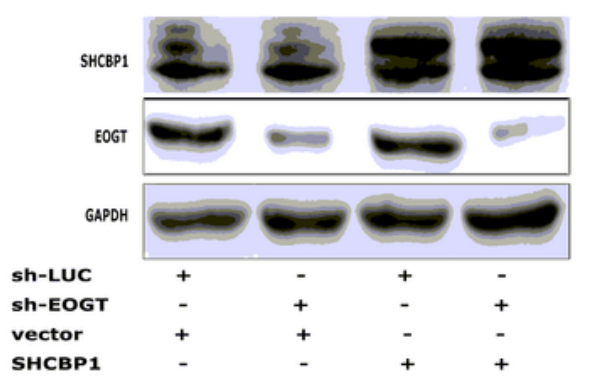

B

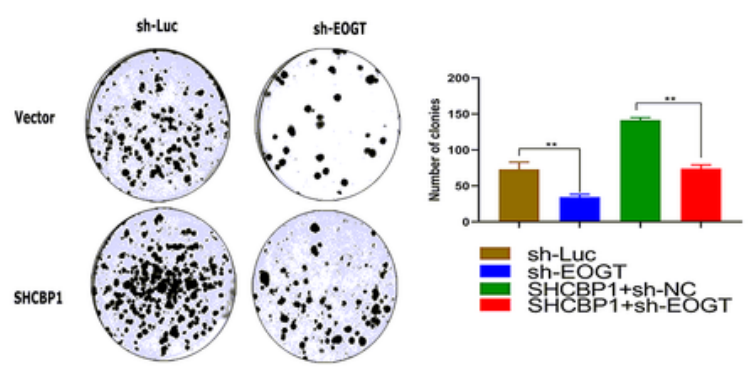

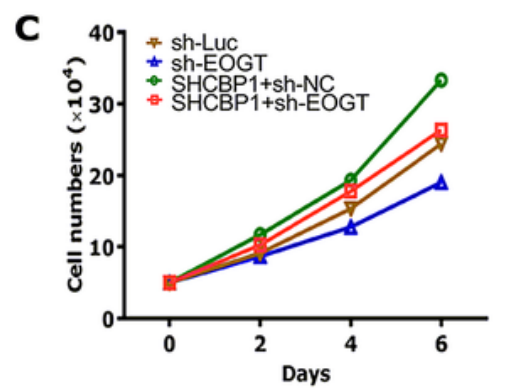

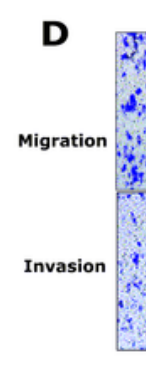

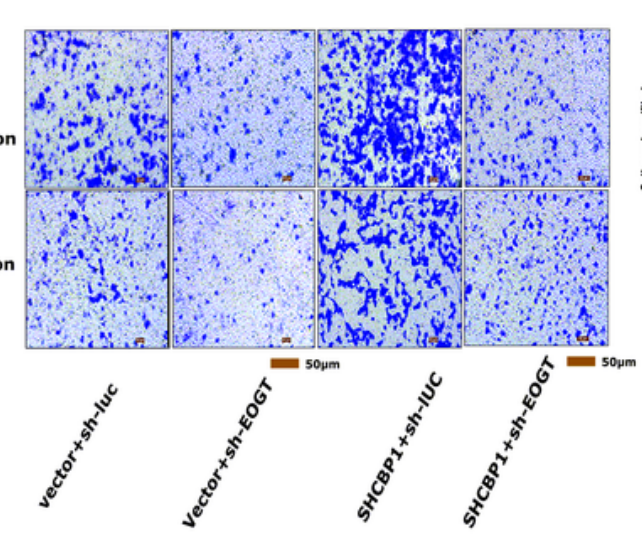

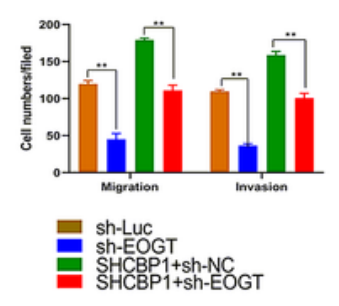

E
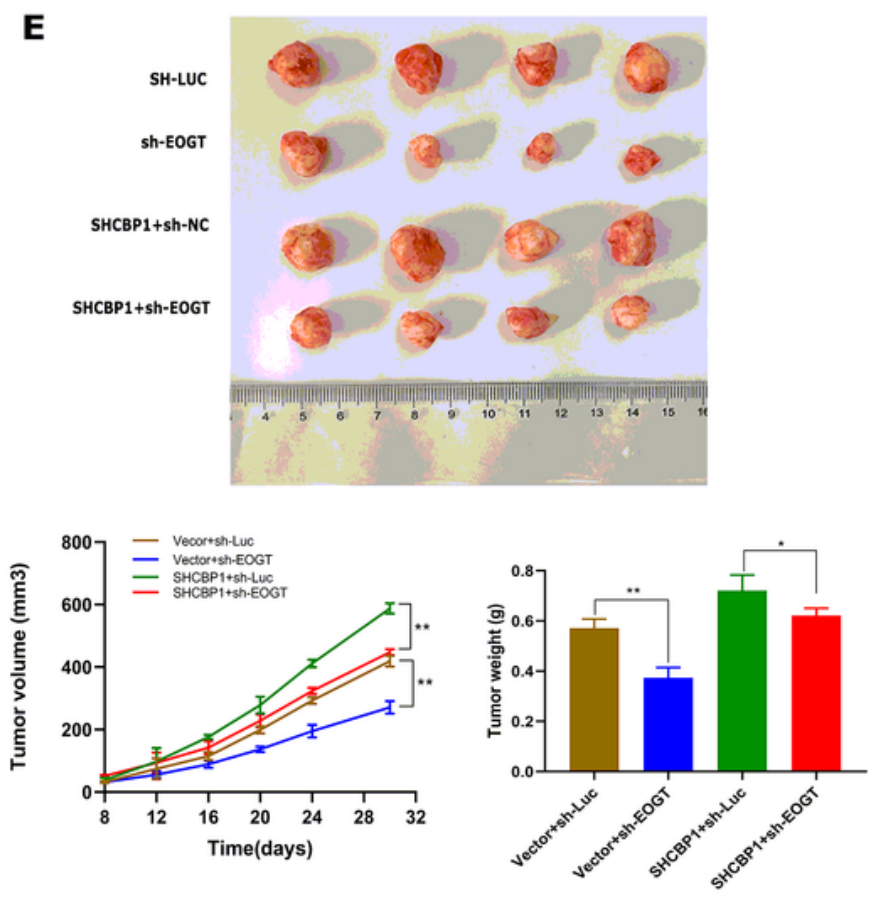

$\mathbf{F}$

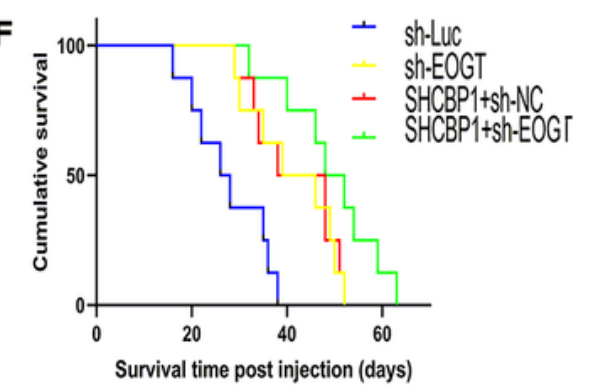

G

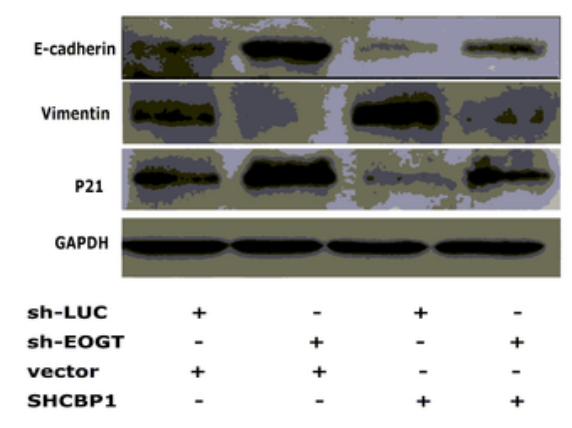

\section{Figure 5}

Knockdown of EOGT inhibits the malignant potential of SHCBP1 in vivo and in vitro. A. SHCBP1overexpressing and vector Panc-1 cellswith stable EOGT knockdown were established. The changes in SHCBP1 and EOGT protein expression were confirmed by Western blotting. B-C.The proliferative ability of different SHCBP1- or EOGT-expressing Panc1 cells was investigated usingCCK-8 and colony formation assays. D.Transwell assays of Panc1 cells with different SHCBP1 or EOGT expression levels were 
performed. Representative images and quantification of migration and invasion are presented $(\star \star \mathrm{P}<0.01$; $\left.{ }^{*} * * \mathrm{P}<0.001\right)$.E. Stable knockdown of EOGT in SHCBP1-overexpressing or vector-transfected cells inhibited PDAC growth in a subcutaneousxenograft model (nude mouse). The tumour volume was measured at the indicated time points $(* \star * P<0.001)$. Tumours were extracted and weighed after the mice were sacrificed $(* \star * P<0.001)$. F.The effect ofEOGT expression on mouse OS afterinjection of Panc-1 cells.G.Western blots forE-cadherin, vimentin, and P21 expressionin Panc-1 cells with varying expression levels of SHCBP1 and EOGT.

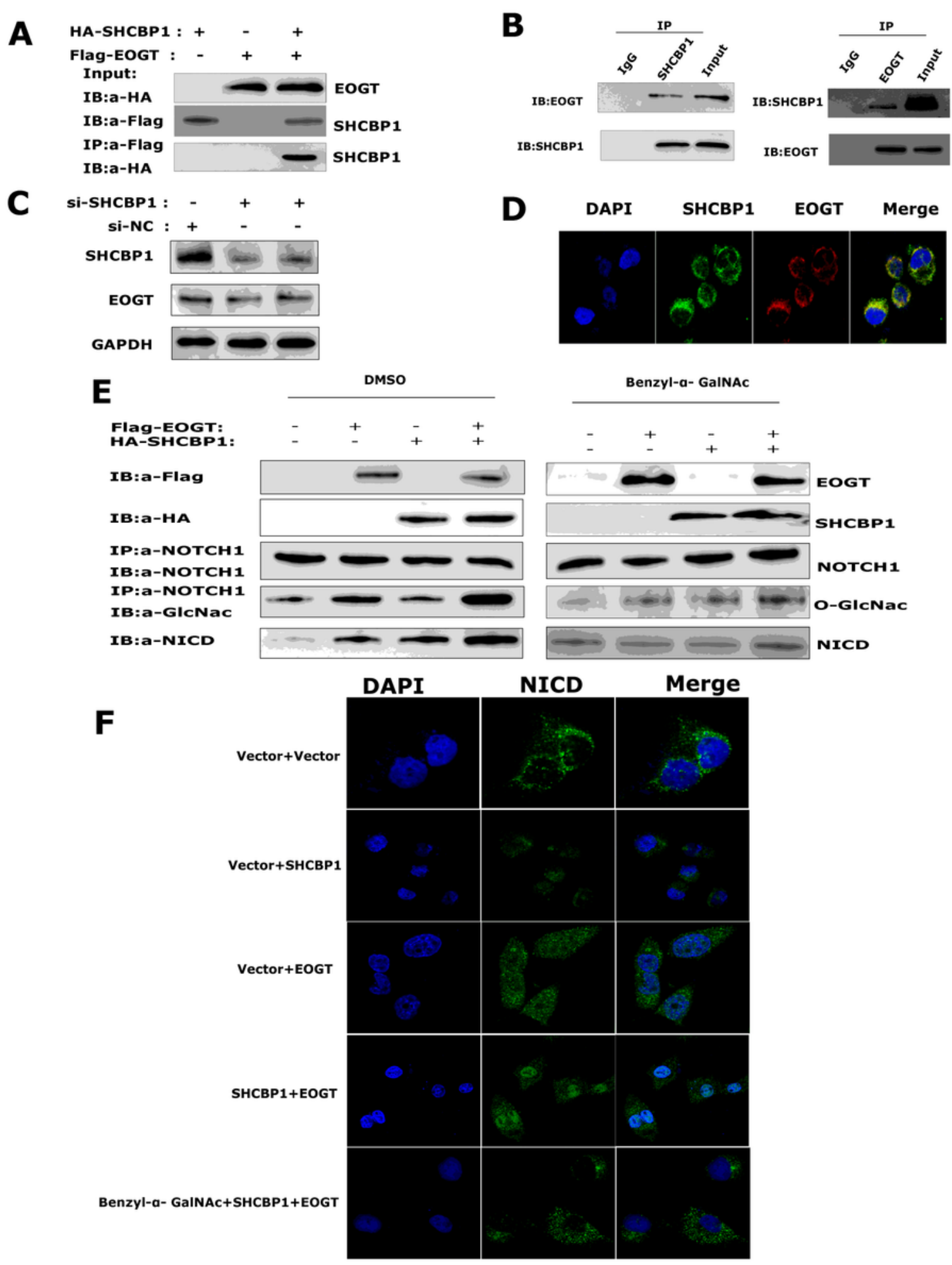




\section{Figure 6}

The SHCBP1-EOGT interactionpromotes NOTCH1 0-GlcNAcylation and promoted nuclear localization of the NICD. A. The interaction between SHCBP1 and EOGT was analysed in HEK-293T cell lysates transfected with plasmids encoding HA-SHCBP1 and Flag-EOGT, proteinswere thenimmunoprecipitated with the corresponding antibodies and subjected to Western blot analysis. B. Endogenous interaction between SHCBP1 and EOGT was assayed in Panc-1 cell lysates via immunoprecipitation with antiSHCBP1 or anti-EOGT antibodies and then Western blot analysis using an anti-EOGT or anti-SHCBP1 antibody, respectively. Ten percent of the input was loaded, and normal IgG was used as a control.C.TheEOGT expression in SHCBP1 knockdown cells.D. Immunofluorescence staining of SHCBP1 (green), EOGT (red) and merge (yellow) in Panc-1 cells.E.Theimpact of O-linked glycosylation of NOTCH1 by SCHBP1 and EOGT on the NICD with or without benzyl-a-GalNAc $(1 \mathrm{mg} / \mathrm{ml})$ was detected by Western blotting.F. Nuclear localizationof the NICD (green) in the presence of EOGT or SHCBP1was tested by immunofluorescence staining. 
A

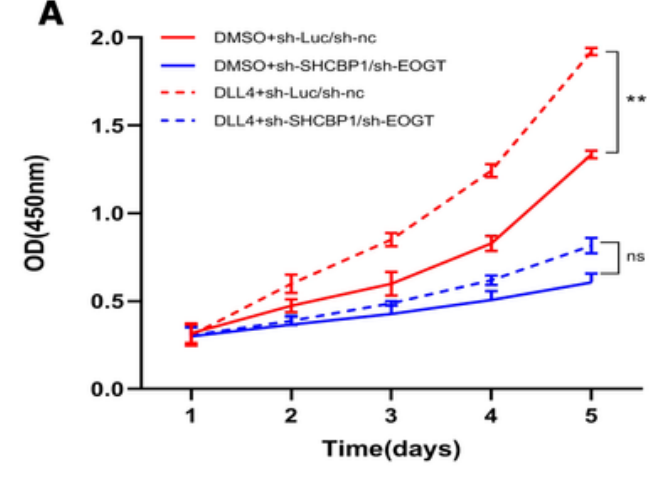

C

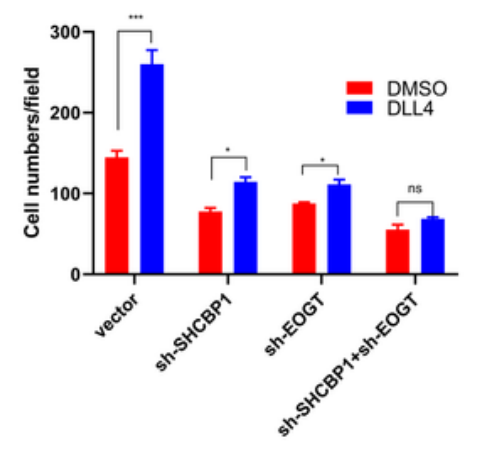

E

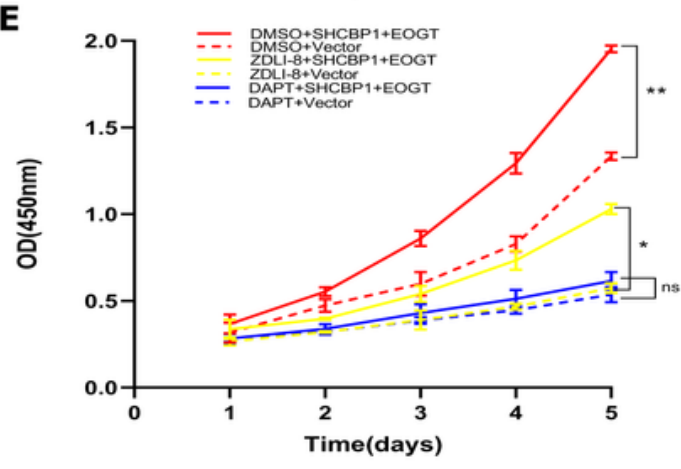

G

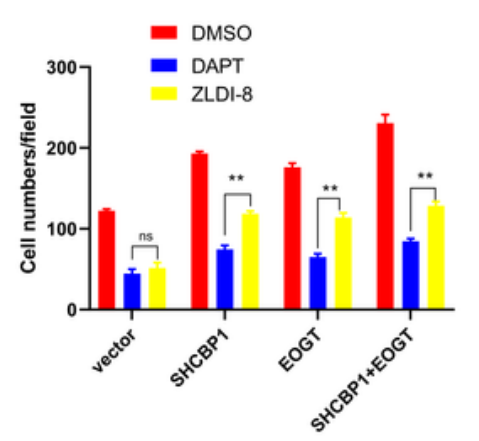

B

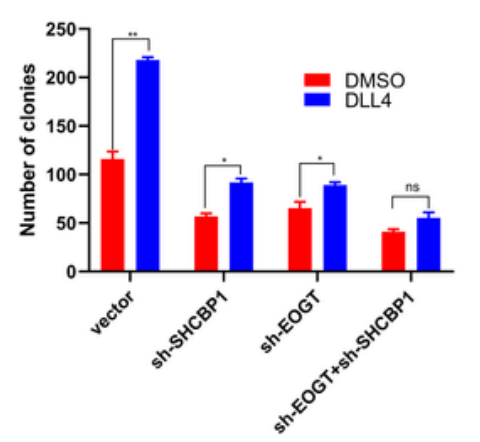

D

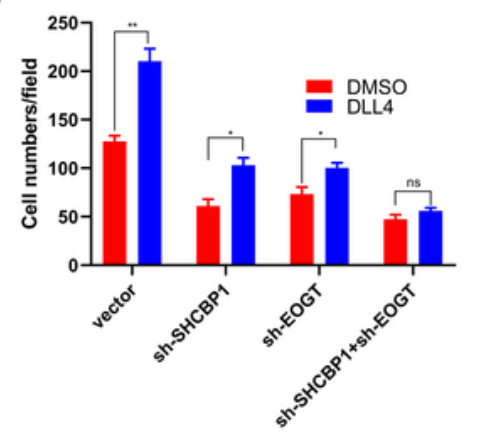

$\mathbf{F}$
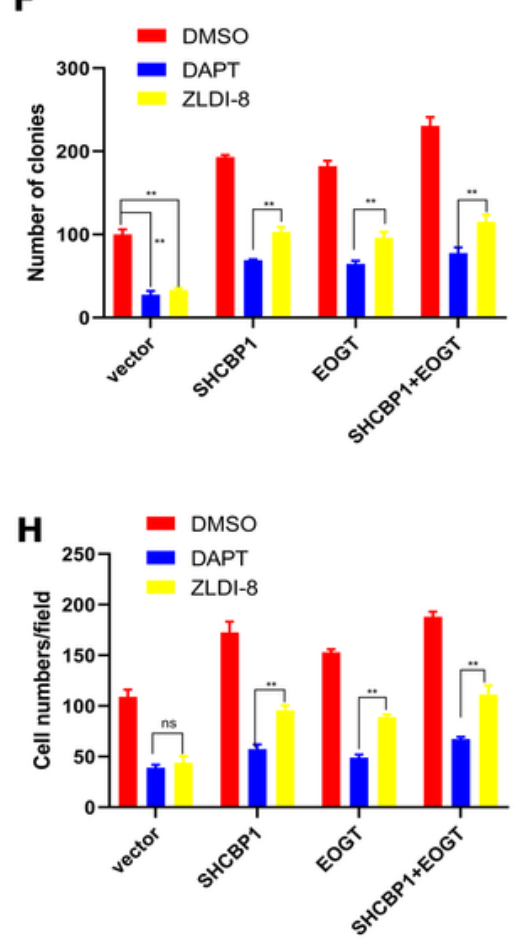

Figure 7

SHCBP1 and EOGT regulate the malignant behaviour of PDAC via the NOTCH1 pathway. A-D. The effect of DLL4 andDMSOon the regulation of proliferation as indicated byCCK8 and cloning assays (A-B) and on migration and invasion as indicated by Transwell assays (C-D). SHCBP1 or/and EOGT knockdown Panc-1 cells. (E-H) The effect of DAPT , ZDLI-8 andDMSOon Panc-1 cell proliferation as indicated by 
CCK8 and cloning assays (E-F) and migration and invasion assays (G-H) Bxpc3 cells with SHCBP1 or/and EOGT overexpression.

A

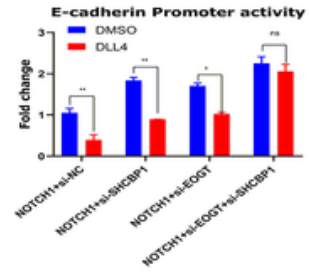

$\mathbf{E}$

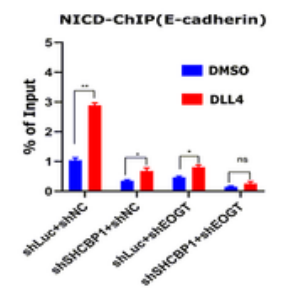

G

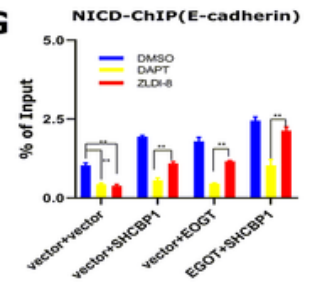

I

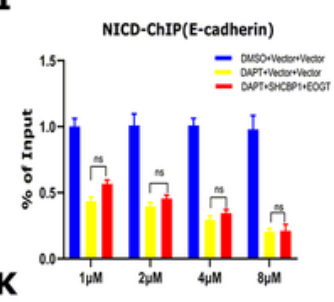

$\mathbf{K}$

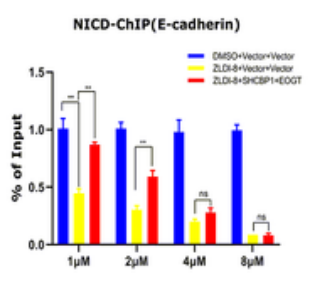

\section{B}
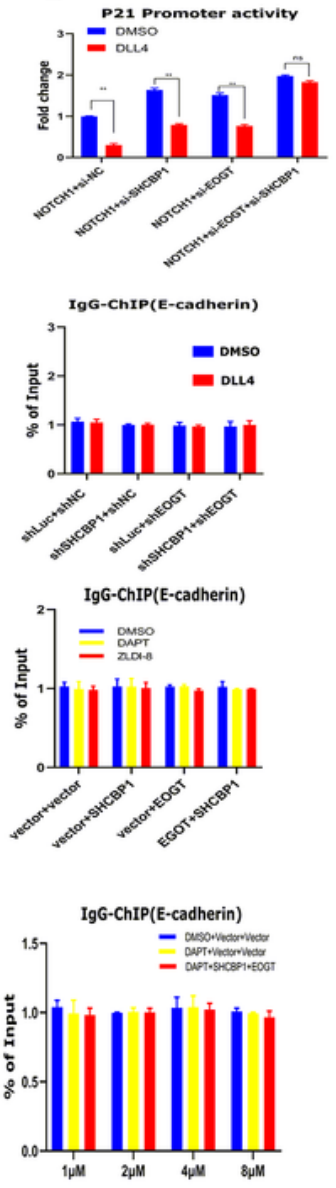

IgG-ChIP(E-cadherin)

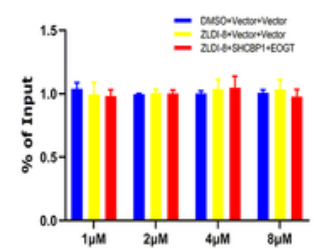

C

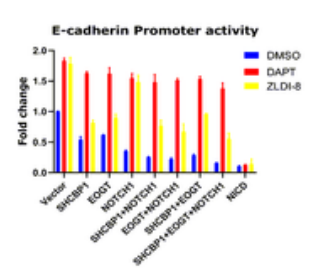

$\mathbf{F}$

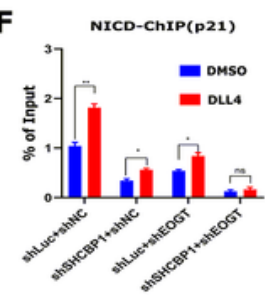

H

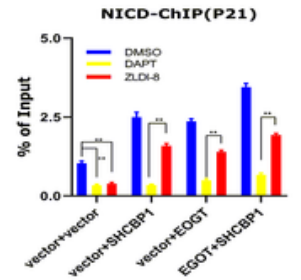

J

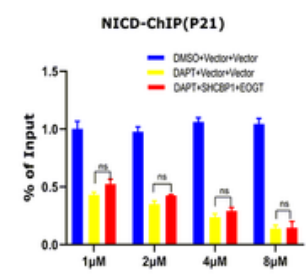

$\mathbf{L}$

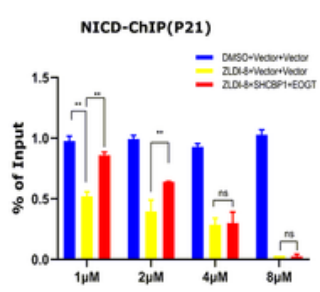

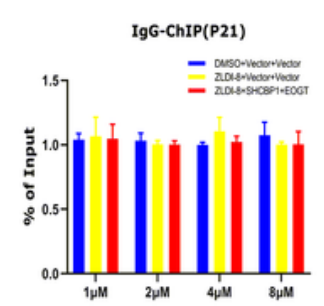
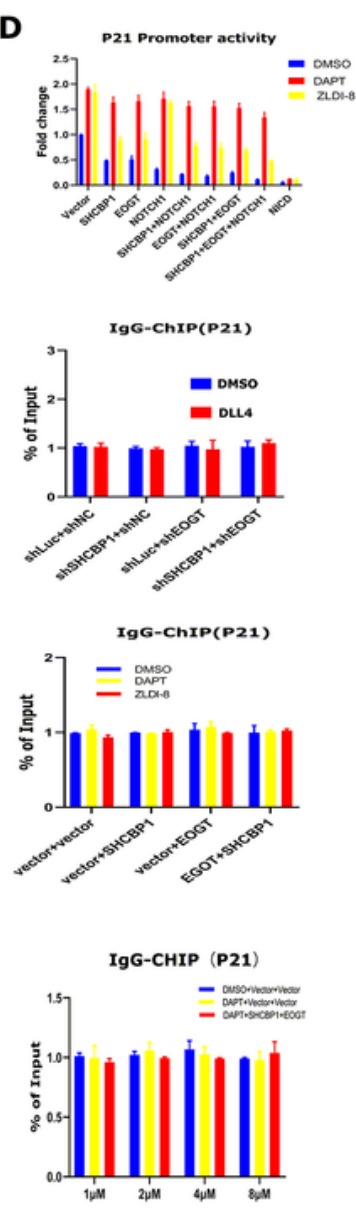

M

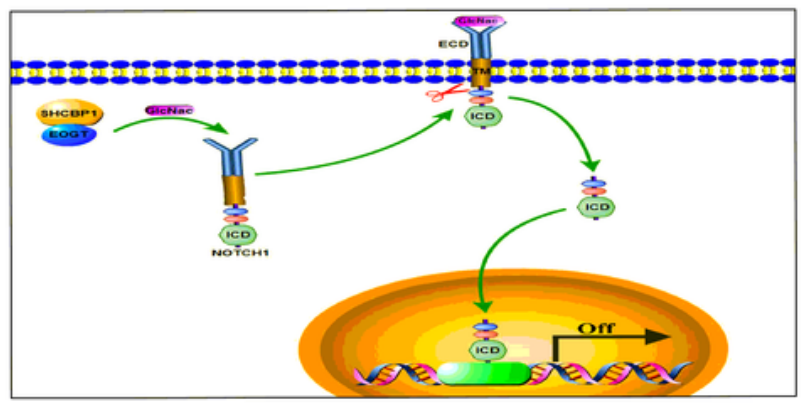

Figure 8

SHCBP1 and EOGT inhibits the transcriptional activity of E-cadherin and P21 by NICD A-D, Luciferase reporter assay. EOGT interacts with SHCBP1 to regulate E-cadherinor P21promoter-driven luciferase activityafter treatment with DLL4 (A, B) or DAPT/ZLDI-8 (C, D). (E-L),ChIP-PCR assay.Panc-1 cells were 
grown and transfected with SHCBP1shRNA, EOGTshRNA, or control shRNA, treated withDMSO or DLL4 and then subjected to ChIP-PCR analysis of the E-cadherin (E) and P21 (F) promoter regions.Bxpc3 cells were grown and transfected with SHCBP1, EOGT, or a control vector, treated withDMSO or DAPT/ZLDI-8 and then subjected to ChIP-PCR analysis of the E-cadherin $(\mathrm{G})$ or P21 $(\mathrm{H})$ promoter regions. $(\mathrm{I}-\mathrm{L})$, Difference concentrations of DAPT/ZLDI- $8(1 \mu \mathrm{M}, 2 \mu \mathrm{M}, 4 \mu \mathrm{M}, 8 \mu \mathrm{M})$ were added, and the data represent the means \pm s.d. of three independent experiments. The antibodies used in the ChIP assay are shown in each panel, and normal IgG was used as a control. The binding activity of each protein is given as a percentage of the total input.M.Model for the SHCBP1 interacting with EOGT enhanced O-GIcNAcylation of $\mathrm{NOTCH} 1$ and promoted the development of pancreatic cancer.

\section{Supplementary Files}

This is a list of supplementary files associated with this preprint. Click to download.

- table1.pdf

- plasmid.docx

- supplementalFigure1.tif

- supplementalFigure2.tif 